TITLE:

\title{
Bioassay Data for Marine Pollution Using Sea Urchin Eggs, 1984 to 1989
}

$\operatorname{AUTHOR}(S)$ :

Kobayashi, Naomasa

\section{CITATION:}

Kobayashi, Naomasa. Bioassay Data for Marine Pollution Using Sea Urchin Eggs, 1984 to 1989. PUBLICATIONS OF THE SETO MARINE BIOLOGICAL LABORATORY 1992, 35(6): 387415

ISSUE DATE:

1992-11-30

URL:

http://hdl.handle.net/2433/176207

RIGHT: 


\title{
Bioassay Data for Marine Pollution Using Sea Urchin \\ Eggs, 1984 to 1989
}

\author{
1984
}

Four experiments were made as follows.

I. Winter season, January 21, using Hemicentrotus pulcherrimus eggs, see Table 1.

II. Spring season, April 3, using H. pulcherrimus eggs, see Table 2.

III. Summer season, June 23, using Anthocidaris crassispina eggs, see Table 3.

IV. Autumn season, September 4, using A. crassispina eggs, see Table 4.

(Notes common to all tables: Fertilization membrane formation examined $3 \mathrm{~min}$. after fertilization; minutes and hours in parentheses respectively after First cleavage, Gastrulation and Pluteus indiacte the time after insemination; the maturation state of gonads used was nearly ripe to full ripe; For the 5 mins. old sperm and 3-6 hrs. old eggs, Degree of inhibitory effect 0 show the non-inhibition, 1 the slight inhibition, 2 the weak, 3 the moderate, 4 the strong and 5 the violent inhibition state of the sea water tested (see Ranking III, Publ. Seto Mar. Biol. Lab., Vol. 30, No. 4/6, p. 224, Table 6, 1985))

\section{5}

Four experiments were made as follows.

I. Winter season, January 24, using Hemicentrotus pulcherrimus eggs, see Table 5.

II. Spring season, March 23, using H. pulcherrimus eggs, see Table 6.

III. Summer season, July 18, using Anthocidaris crassispina eggs, see Table 7.

IV. Autumn season, August 28, using A. crassispina eggs, see Table 8.

(Notes common to all tables: See the notes mentioned above.)

1986

Three experiments were made as follows.

I. Winter season, January 23, using Hemicentrotus pulcherrimus eggs, see Table 9.

II. Spring season, March 28, using H. pulcherrimus eggs, see Table 10.

III. Autumn season, September 4, using Anthocidaris crassisfina eggs, sec Table 11. (Notes common to all tables: See the notes mentioned above.)

Four experiments were made as follows.

I. Winter season, January 17, using Hemicentrotus pulcherrimus eggs, see Table 12.

II. Spring season, March 19, H. pulcherrimus eggs, see Table 13.

I1I. Summer season, June 10, using Anthocidaris crassispina eggs, see Table 14.

Publ. Seto Mar. Biol. Lab., 35(6), 387 415, $1992 . \quad$ (Biological Data 2) 
IV. Autumn season, August 28, using A. crassispina eggs, see Table 15.

(Notes common to all tables: See the notes mentoined above.)

1988

Four experiments were made as follows.

I. Spring season, March 29, using Hemicentrotus pulcherrimus eggs, see Table 16 and May 30, using Anthocidaris crassispina eggs, see Table 17.

II. Summer season, July 19 and August 19, using A. crassispina eggs, see Tables 18 and 19 .

(Notes common to all tables: See the notes mentioned above; For the 0 mins. old sperm and 0 hrs. old eggs, Degree of inhibitory effect 0 show the no inhibition, 1 the weak, 2 the moderate and 3 the strong inhibition state of the sea water tested (see Ranking IV, Publ. Seto Mar. Biol. Lab., Vol. 34, Nos. 4/6, p. 235, Table 8, 1990))

1989

Four experiments were made as follows.

I. Winter season, February 21, using Hemicentrotus pulcherrimus eggs, see Table 20.

II. Spring season, April 5, using H. pulcherrimus eggs, see Table 21.

III. Summer season, July 5, using Anthocidaris crassispina eggs, see Table 22.

IV. Autumn season, August 30, using A. crassispina eggs, see Table 23.

(Notes common to all tables: See the notes mentioned above.)

Naomasa Kobayashi

Biological Laboratory Doshisha University Kyoto 602, Japan 
Table 1. Results of the Jan. 21, 1984 experiment with eggs of Hemicentrotus pulcherrimus. Wind; 0, Test water temperature; $18^{\circ} \mathrm{C}, 5$ mins. old sperms, 6 hrs. old eggs. Ranking III 1985.

\begin{tabular}{|c|c|c|c|c|c|c|c|c|c|c|}
\hline \multirow{3}{*}{$\begin{array}{l}\text { Location } \\
\qquad \begin{array}{l}\text { (depth) }\end{array}\end{array}$} & \multicolumn{9}{|c|}{ Time after insemination } & \multirow{3}{*}{$\begin{array}{l}\text { Degree of } \\
\text { inhibitory } \\
\text { effect }\end{array}$} \\
\hline & \multirow{2}{*}{ 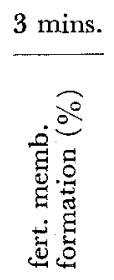 } & \multicolumn{3}{|c|}{75 mins. } & \multicolumn{3}{|c|}{$22 \mathrm{hrs}$} & \multicolumn{2}{|c|}{$45 \mathrm{hrs}}$. & \\
\hline & & 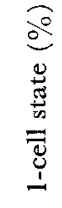 & 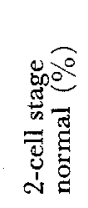 & 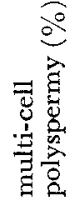 & 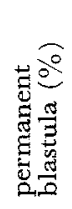 & 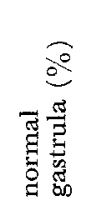 & 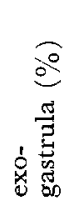 & 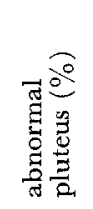 & 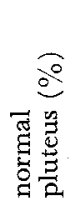 & \\
\hline \multicolumn{11}{|l|}{$(\mathrm{m})$} \\
\hline \multirow{3}{*}{$\begin{array}{l}\text { Running } \\
\text { sea water of } \\
\text { Laboratory }\end{array}$} & 99.5 & 0.5 & 99.5 & 0 & 0.5 & 99.5 & 0 & 1.0 & 99.0 & \\
\hline & 98.5 & 1.0 & 99.0 & 0 & 1.0 & 99.0 & 0 & 1.5 & 98.5 & 0 \\
\hline & 99.0 & 1.0 & 99.0 & 0 & 0.5 & 99.5 & 0 & 1.5 & 98.5 & \\
\hline \multirow{3}{*}{$\begin{array}{l}\text { Water from } \\
\text { open sea side } \\
\text { of Hatakejima } \\
\text { Surface }\end{array}$} & 99.0 & 1.0 & 99.0 & 0 & 0.5 & 99.5 & 0 & 1.0 & 99.0 & \\
\hline & 99.5 & 0.5 & 99.5 & 0 & 0.5 & 99.5 & 0 & 0.5 & 99.5 & 0 \\
\hline & 99.5 & 1.0 & 99.0 & 0 & 1.0 & 99.0 & 0 & 1.5 & 98.5 & \\
\hline \multirow{4}{*}{$\begin{array}{l}\text { Water from } \\
\text { land side of } \\
\text { Hatakejima } \\
\text { Surface }\end{array}$} & 96.5 & 4.0 & 96.0 & 0 & 3.5 & 96.5 & 0 & 5.5 & 94.5 & \\
\hline & 98.0 & 2.5 & 97.5 & 0 & 2.5 & 97.5 & 0 & 6.0 & 94.0 & 1 \\
\hline & 97.0 & 3.5 & 96.5 & 0 & 3.0 & 97.0 & 0 & 4.5 & 95.5 & \\
\hline & 95.0 & 6.0 & 94.0 & 0 & 3.5 & 96.5 & 0 & 5.5 & 94.5 & \\
\hline \multirow[t]{2}{*}{ Bottom (7) } & 98.0 & 2.5 & 97.5 & 0 & 4.0 & 96.0 & 0 & 6.5 & 93.5 & 1 \\
\hline & 96.0 & 5.0 & 95.0 & 0 & 3.0 & 97.0 & 0 & 5.0 & 95.0 & \\
\hline \multirow{3}{*}{$\begin{array}{l}\text { Sea water from } \\
\text { Tsunashirazu } \\
\text { cove } \\
\text { Surface }\end{array}$} & 92.0 & 9.0 & 91.0 & 0 & 56.5 & 43.5 & 0 & 29.5 & 70.5 & \\
\hline & 96.5 & 4.5 & 95.5 & 0 & 52.0 & 48.0 & 0 & 27.0 & 73.0 & 5 \\
\hline & 94.0 & 6.5 & 93.5 & 0 & 50.5 & 49.5 & 0 & 24.0 & 76.0 & \\
\hline \multirow{3}{*}{ Bottom (5) } & 94.5 & 7.0 & 93.0 & 0 & 22.0 & 78.0 & 0 & 22.0 & 78.0 & \\
\hline & 96.0 & 5.5 & 94.5 & 0 & 19.0 & 81.0 & 0 & 21.0 & 79.0 & 4 \\
\hline & 93.0 & 7.5 & 92.5 & 0 & 18.0 & 82.0 & 0 & 24.5 & 75.5 & \\
\hline
\end{tabular}


Table 2. Results of the Apr. 3, 1984 experiment with eggs of Hemicentrotus pulcherrimus.

Wind; 0, Test water temperature; $18^{\circ} \mathrm{C}, 5$ mins. old sperms, 6 hrs. old eggs. Ranking III 1985.

\begin{tabular}{|c|c|c|c|c|c|c|c|c|c|c|}
\hline \multirow{3}{*}{ Location } & \multicolumn{9}{|c|}{ Time after insemination } & \multirow{3}{*}{$\begin{array}{l}\text { Degree of } \\
\text { inhibitory } \\
\text { effect }\end{array}$} \\
\hline & \multirow{2}{*}{ 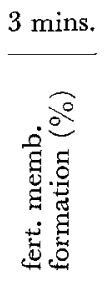 } & \multicolumn{3}{|c|}{75 mins. } & \multicolumn{3}{|c|}{24 hrs. } & \multicolumn{2}{|c|}{$48 \mathrm{hrs}}$. & \\
\hline & & 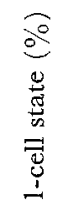 & 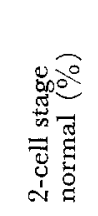 & 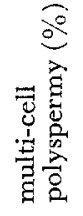 & 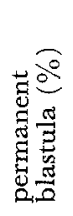 & 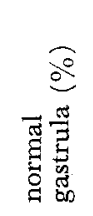 & $\frac{20}{3}$ & 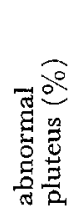 & 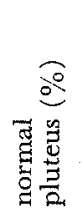 & \\
\hline \multicolumn{11}{|l|}{ (m) } \\
\hline \multirow{3}{*}{$\begin{array}{l}\text { Running } \\
\text { sea water of } \\
\text { Laboratory }\end{array}$} & 99.0 & 1.5 & 98.5 & 0 & 0.5 & 99.5 & 0 & 1.5 & 98.5 & \\
\hline & 99.5 & 1.0 & 99.0 & 0 & 1.0 & 99.0 & 0 & 1.0 & 99.0 & 0 \\
\hline & 98.0 & 2.0 & 98.0 & 0 & 0.5 & 99.5 & 0 & 1.5 & 98.5 & \\
\hline \multirow{3}{*}{$\begin{array}{l}\text { Water from } \\
\text { open sea side } \\
\text { of Hatakejima } \\
\text { Surface }\end{array}$} & 99.5 & 1.0 & 99.0 & 0 & 0.5 & 99.5 & 0 & 1.0 & 99.0 & \\
\hline & 99.0 & 1.5 & 98.5 & 0 & 0.5 & 99.5 & 0 & 0.5 & 99.5 & 0 \\
\hline & 98.0 & 2.0 & 98.0 & 0 & 1.0 & 99.0 & 0 & 1.5 & 98.5 & \\
\hline \multirow{4}{*}{$\begin{array}{l}\text { Water from } \\
\text { land side of } \\
\text { Hatakejima } \\
\text { Surface }\end{array}$} & 97.5 & 3.0 & 97.0 & 0 & 1.0 & 99.0 & 0 & 1.5 & 98.5 & \\
\hline & 97.0 & 3.5 & 96.5 & 0 & 1.5 & 98.5 & 0 & 1.0 & 99.0 & 0 \\
\hline & 98.5 & 2.0 & 98.0 & 0 & 3.5 & 96.5 & 0 & 2.5 & 97.5 & \\
\hline & 97.0 & 4.0 & 96.0 & 0 & 1.5 & 98.5 & 0 & 2.0 & 98.0 & \\
\hline \multirow[t]{2}{*}{ Bottom (7) } & 96.0 & 4.5 & 95.5 & 0 & 3.0 & 97.0 & 0 & 2.5 & 97.5 & 0 \\
\hline & 98.0 & 3.0 & 97.0 & 0 & 2.5 & 97.5 & 0 & 4.0 & 96.0 & \\
\hline \multirow{3}{*}{$\begin{array}{l}\text { Sea water from } \\
\text { Tsunashirazu } \\
\text { cove } \\
\text { Surface }\end{array}$} & 98.0 & 2.5 & 97.5 & 0 & 1.5 & 98.5 & 0 & 6.0 & 94.0 & \\
\hline & 97.0 & 4.5 & 95.5 & 0 & 3.0 & 97.0 & 0 & 5.5 & 94.5 & 1 \\
\hline & 96.5 & 4.0 & 96.0 & 0 & 2.0 & 98.0 & 0 & 7.0 & 93.0 & \\
\hline \multirow{3}{*}{ Bottom (5) } & 96.0 & 4.0 & 96.0 & 0 & 2.5 & 97.5 & 0 & 8.0 & 92.0 & \\
\hline & 97.5 & 5.0 & 95.0 & 0 & 3.5 & 96.5 & 0 & 7.5 & 92.5 & 1 \\
\hline & 97.0 & 3.5 & 96.5 & 0 & 2.0 & 98.0 & 0 & 6.0 & 94.0 & \\
\hline
\end{tabular}


Table 3. Results of the June 23, 1984, experiment with eggs of Anthocidaris crassispina.

Wind; 0, Test water temperature; $26^{\circ} \mathrm{C}, 5$ mins. old sperms, 3 hrs. old eggs. Ranking III 1985.

\begin{tabular}{|c|c|c|c|c|c|c|c|c|c|c|}
\hline \multirow{3}{*}{$\begin{array}{c}\text { Location } \\
\qquad \\
\text { (depth) }\end{array}$} & \multicolumn{9}{|c|}{ Time after insemination } & \multirow{3}{*}{$\begin{array}{l}\text { Degree of } \\
\text { inhibitory } \\
\text { effect }\end{array}$} \\
\hline & \multirow{2}{*}{$\frac{3 \text { mins. }}{\text { 20 }}$} & \multicolumn{3}{|c|}{60 mins. } & \multicolumn{3}{|c|}{$16 \mathrm{hrs}$. } & \multicolumn{2}{|c|}{$26 \mathrm{hrs}}$. & \\
\hline & & 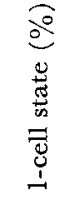 & 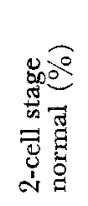 & 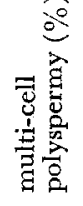 & 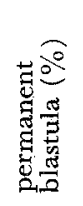 & 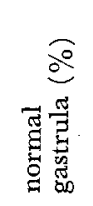 & 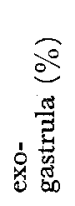 & 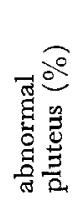 & 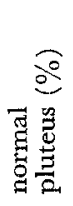 & \\
\hline \multicolumn{11}{|l|}{$(\mathrm{m})$} \\
\hline \multirow{3}{*}{$\begin{array}{l}\text { Running } \\
\text { sea water of } \\
\text { Laboratory }\end{array}$} & 99.0 & 1.5 & 98.5 & 0 & 0.5 & 99.5 & 0 & 1.0 & 99.0 & \\
\hline & 99.5 & 1.0 & 99.0 & 0 & 0 & 100 & 0 & 0.5 & 99.5 & 0 \\
\hline & 98.5 & 2.0 & 98.0 & 0 & 1.0 & 99.0 & 0 & 1.0 & 99.0 & \\
\hline \multirow{4}{*}{$\begin{array}{l}\text { Water from } \\
\text { open sea side } \\
\text { of Hatakejima } \\
\text { Surface }\end{array}$} & 98.5 & 1.5 & 98.5 & 0 & 0.5 & 99.5 & 0 & 0.5 & 99.5 & \\
\hline & 99.0 & 1.0 & 99.0 & 0 & 1.0 & 99.0 & 0 & 1.5 & 98.5 & 0 \\
\hline & 99.5 & 1.0 & 99.0 & 0 & 0.5 & 99.5 & 0 & 1.0 & 99.0 & \\
\hline & 77.0 & 24.5 & 75.5 & 0 & 14.5 & 85.5 & 0 & $14 \cdot 5$ & 85.5 & \\
\hline \multirow[t]{2}{*}{ Bottom (15) } & 76.5 & 26.0 & 74.0 & 0 & 12.5 & 87.5 & 0 & 16.0 & 84.0 & 3 \\
\hline & 71.0 & 30.0 & 70.0 & 0 & 13.0 & 87.0 & 0 & 13.0 & 87.0 & \\
\hline \multirow{4}{*}{$\begin{array}{l}\text { Water from } \\
\text { land side of } \\
\text { Hatakejima } \\
\text { Surface }\end{array}$} & 76.5 & 26.0 & 74.0 & 0 & 14.0 & 86.0 & 0 & 16.0 & 84.0 & \\
\hline & 78.0 & 25.0 & 75.0 & 0 & 11.0 & 89.0 & 0 & 17.0 & 83.0 & 3 \\
\hline & 72.0 & 30.0 & 70.0 & 0 & 15.5 & 84.5 & 0 & 13.0 & 87.0 & \\
\hline & 81.0 & 19.5 & 80.5 & 0 & 1.0 & 99.0 & 0 & 3.0 & 97.0 & \\
\hline \multirow[t]{2}{*}{ Bottom (7) } & 79.0 & 23.5 & 76.5 & 0 & 4.0 & 96.0 & 0 & 3.5 & 96.6 & 2 \\
\hline & 75.5 & 27.0 & 73.0 & 0 & 4.5 & 95.5 & 0 & 6.5 & 93.5 & \\
\hline \multirow{3}{*}{$\begin{array}{l}\text { Sea water from } \\
\text { Tsunashirazu } \\
\text { cove } \\
\text { Surface }\end{array}$} & 78.5 & 24.0 & 76.0 & 0 & 11.0 & 89.0 & 0 & 15.0 & 85.0 & \\
\hline & 73.0 & 28.0 & 72.0 & 0 & 16.5 & 83.5 & 0 & 15.5 & 84.5 & 3 \\
\hline & 71.5 & 31.5 & $68 \cdot 5$ & 0 & 13.0 & 87.0 & 0 & 17.0 & 83.0 & \\
\hline \multirow{3}{*}{ Bottom (5) } & 76.0 & 25.0 & 75.0 & 0 & 13.0 & 87.0 & 0 & 16.0 & 84.0 & \\
\hline & 70.0 & 32.0 & 68.0 & 0 & 18.5 & 81.5 & 0 & 13.5 & 86.5 & 3 \\
\hline & 68.5 & 33.0 & 67.0 & 0 & 16.0 & 84.0 & 0 & 19.0 & 81.0 & \\
\hline
\end{tabular}


Table 4. Results of the Sep. 4, 1984, experiment with eggs of Anthocidaris crassispina.

Wind; 0, Test water temperature; $27^{\circ} \mathrm{C}, 5$ mins. old sperms, 3 hrs. old eggs. Ranking III 1985.

\begin{tabular}{|c|c|c|c|c|c|c|c|c|c|c|}
\hline \multirow{3}{*}{ Location } & \multicolumn{9}{|c|}{ Time after insemination } & \multirow{3}{*}{$\begin{array}{l}\text { Degree of } \\
\text { inhibitory } \\
\text { effect }\end{array}$} \\
\hline & \multirow{2}{*}{ 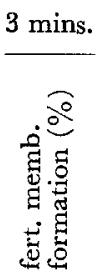 } & \multicolumn{3}{|c|}{60 mins. } & \multicolumn{3}{|c|}{$15 \mathrm{hrs}}$. & \multicolumn{2}{|c|}{24 hrs. } & \\
\hline & & 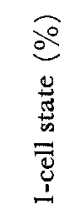 & 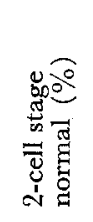 & 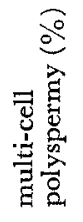 & 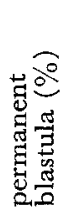 & 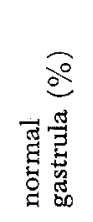 & 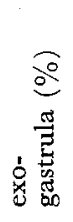 & 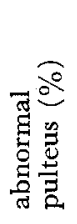 & 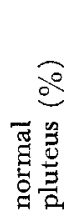 & \\
\hline \multicolumn{11}{|l|}{$(\mathrm{m})$} \\
\hline \multirow{3}{*}{$\begin{array}{l}\text { Running } \\
\text { sea water of } \\
\text { Laboratory }\end{array}$} & 99.5 & 1.0 & 99.0 & 0 & 0.5 & 99.5 & 0 & 1.0 & 99.0 & \\
\hline & 99.0 & 1.5 & 98.5 & 0 & 1.0 & 99.0 & 0 & 0.5 & 99.5 & 0 \\
\hline & 99.0 & 1.0 & 99.0 & 0 & 1.0 & 99.0 & 0 & 2.0 & 98.0 & \\
\hline \multirow{4}{*}{$\begin{array}{l}\text { Water from } \\
\text { open sea side } \\
\text { of Hatakejima } \\
\text { Surface }\end{array}$} & 98.0 & 2.0 & 98.0 & 0 & 2.0 & 98.0 & 0 & 4.0 & 96.0 & \\
\hline & 99.5 & 1.5 & 98.5 & 0 & 2.5 & 97.5 & 0 & 3.5 & 96.5 & 1 \\
\hline & 99.0 & 2.0 & 98.0 & 0 & 1.5 & 98.5 & 0 & 6.0 & 94.0 & \\
\hline & 98.0 & 2.5 & 97.5 & 0 & 1.0 & 99.0 & 0 & 1.5 & 98.5 & \\
\hline \multirow[t]{2}{*}{ Bottom (15) } & 99.0 & 1.5 & 98.5 & 0 & 2.5 & 97.5 & 0 & 2.0 & 98.0 & 0 \\
\hline & 98.0 & 2.0 & 98.0 & 0 & 1.0 & 99.0 & 0 & 1.5 & 98.5 & \\
\hline \multirow{3}{*}{$\begin{array}{l}\text { Water from } \\
\text { land side of } \\
\text { Hatakejima } \\
\text { Surface }\end{array}$} & 98.5 & 2.0 & 98.0 & 0 & 1.5 & 98.5 & 0 & 2.0 & 98.0 & \\
\hline & 98.0 & 2.5 & 97.5 & 0 & 2.0 & 98.0 & 0 & 1.5 & 98.5 & 0 \\
\hline & 98.0 & 3.0 & 97.0 & 0 & 1.0 & 99.0 & 0 & 2.5 & 97.5 & \\
\hline \multirow{3}{*}{ Bottom (7) } & 98.0 & 2.5 & 97.5 & 0 & 2.0 & 98.0 & 0 & 5.5 & 94.5 & \\
\hline & 98.0 & 2.0 & 98.0 & 0 & 2.5 & 97.5 & 0 & 4.0 & 96.0 & 1 \\
\hline & 97.5 & 3.0 & 97.0 & 0 & 2.0 & 98.0 & 0 & 3.5 & 96.5 & \\
\hline \multirow{3}{*}{$\begin{array}{l}\text { Sea water from } \\
\text { Tsunashirazu } \\
\text { cove } \\
\text { Surface }\end{array}$} & 99.0 & 2.0 & 98.0 & 0 & 1.5 & 98.5 & 0 & 1.5 & 98.5 & \\
\hline & 98.0 & 2.5 & 97.5 & 0 & 2.0 & 98.0 & 0 & 2.0 & 98.0 & 0 \\
\hline & 96.5 & 5.0 & 95.0 & 0 & 1.0 & $99 \cdot 0$ & 0 & 3.5 & 96.5 & \\
\hline \multirow{3}{*}{ Bottom (5) } & 93.0 & 8.0 & 92.0 & 0 & 1.5 & 98.5 & 0 & 10.5 & 89.5 & \\
\hline & 87.0 & 13.5 & 86.5 & 0 & 3.0 & 97.0 & 0 & 8.5 & 91.5 & 2 \\
\hline & 89.0 & 12.0 & 88.0 & 0 & 4.0 & 96.0 & 0 & 12.0 & 88.0 & \\
\hline
\end{tabular}


Table 5. Results of the Jan. 24, 1985 experiment with eggs of Hemicontrotus pulcherrimus.

Wind; NW1, Test water temperature; $18^{\circ} \mathrm{C}, 5$ mins. old sperms, 6 hrs. old eggs. Ranking III 1985.

\begin{tabular}{|c|c|c|c|c|c|c|c|c|c|c|}
\hline \multirow{3}{*}{$\begin{array}{l}\text { Location } \\
\qquad \begin{array}{r}\text { (depth) }\end{array}\end{array}$} & \multicolumn{9}{|c|}{ Time after insemination } & \multirow{3}{*}{$\begin{array}{l}\text { Degree of } \\
\text { inhibitory } \\
\text { effect }\end{array}$} \\
\hline & \multirow{2}{*}{$\frac{3 \text { mins. }}{\text { कृ }}$} & \multicolumn{3}{|c|}{75 mins. } & \multicolumn{3}{|c|}{23 hrs. } & \multicolumn{2}{|c|}{$45 \mathrm{hrs}$. } & \\
\hline & & 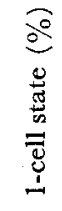 & 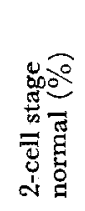 & 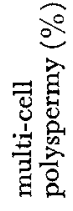 & 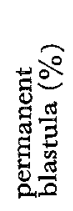 & 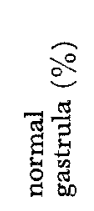 & $\frac{2}{2}$ & 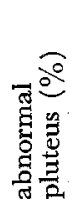 & 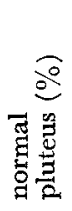 & \\
\hline \multicolumn{11}{|l|}{ (m) } \\
\hline \multirow{2}{*}{$\begin{array}{l}\text { Running } \\
\text { sea water of } \\
\text { Laboratory }\end{array}$} & 98.5 & 2.0 & 98.0 & 0 & 0.5 & 99.5 & 0 & 1.5 & 98.5 & \\
\hline & 99.0 & 1.5 & 98.5 & 0 & 0.5 & 99.5 & 0 & 0.5 & 99.5 & 0 \\
\hline \multirow{2}{*}{$\begin{array}{l}\text { Water from } \\
\text { open sea side } \\
\text { of Hatakejima } \\
\text { Surface }\end{array}$} & 99.0 & 2.0 & 98.0 & 0 & 0 & 100 & 0 & 1.0 & 99.0 & \\
\hline & 98.0 & 1.5 & 98.5 & 0 & 0.5 & 99.5 & 0 & 0.5 & 99.5 & 0 \\
\hline \multirow{2}{*}{$\begin{array}{l}\text { Water from } \\
\text { land side of } \\
\text { Hatakejima } \\
\text { Surface }\end{array}$} & 97.0 & 3.0 & 97.0 & 0 & 1.5 & 98.5 & 0 & 2.5 & 97.5 & \\
\hline & 98.0 & 3.5 & 96.5 & 0 & 1.0 & 99.0 & 0 & 2.0 & 98.0 & 0 \\
\hline \multirow{2}{*}{ Bottom (7) } & 96.5 & 4.0 & 96.0 & 0 & 3.0 & 97.0 & 0 & 7.5 & 92.5 & \\
\hline & 97.0 & 5.0 & 95.0 & 0 & 2.0 & 98.0 & 0 & 6.5 & 93.5 & 1 \\
\hline \multirow{2}{*}{$\begin{array}{l}\text { Sea water from } \\
\text { Tsunashirazu } \\
\text { cove } \\
\text { Surface }\end{array}$} & 98.0 & 3.5 & 96.5 & 0 & 2.0 & 98.0 & 0 & 8.0 & 92.0 & \\
\hline & 97.5 & 4.0 & 96.0 & 0 & 1.5 & 98.5 & 0 & 5.5 & 94.5 & 1 \\
\hline \multirow{2}{*}{ Bottom (5) } & 93.0 & 9.0 & 90.5 & 0.5 & 52.5 & 47.5 & 0 & 83.5 & 16.5 & \\
\hline & 92.5 & 8.5 & 90.5 & 1.0 & 60.5 & 39.5 & 0 & 58.0 & 42.0 & 5 \\
\hline
\end{tabular}


Table 6. Results of the Mar. 23, 1985 experiment with eggs of Hemicentrotus pulcherrimus.

Wind; 0, Test water temperature; $17^{\circ} \mathrm{C}, 5$ mins. old sperms, 6 hrs. old eggs. Ranking III 1995.

\begin{tabular}{|c|c|c|c|c|c|c|c|c|c|c|}
\hline \multirow{3}{*}{ Location } & \multicolumn{9}{|c|}{ Time after insemination } & \multirow{3}{*}{$\begin{array}{l}\text { Degree of } \\
\text { inhibotory } \\
\text { effect }\end{array}$} \\
\hline & \multirow{2}{*}{ 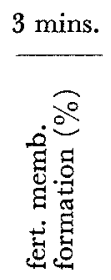 } & \multicolumn{3}{|c|}{75 mins. } & \multicolumn{3}{|c|}{$24 \mathrm{hrs}$. } & \multicolumn{2}{|c|}{48 hrs. } & \\
\hline & & 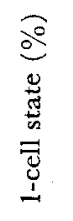 & 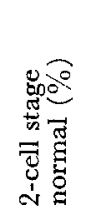 & 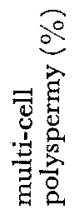 & 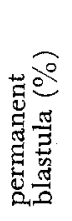 & 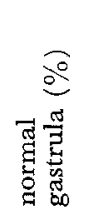 & $\frac{20}{20}$ & 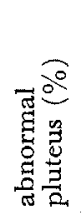 & 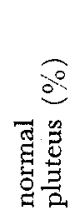 & \\
\hline \multicolumn{11}{|l|}{$(\mathrm{m})$} \\
\hline \multirow{3}{*}{$\begin{array}{l}\text { Running } \\
\text { sea water of } \\
\text { Laboratory }\end{array}$} & 96.5 & 4.5 & 95.0 & 0.5 & 1.0 & 99.0 & 0 & 0.5 & 99.5 & \\
\hline & 94.5 & 6.0 & 94.0 & 0 & 0.5 & 99.5 & 0 & 0.5 & 99.5 & 0 \\
\hline & 98.0 & 2.0 & 98.0 & 0 & 0.5 & 99.5 & 0 & 1.0 & 99.0 & \\
\hline \multirow{3}{*}{$\begin{array}{l}\text { Water from } \\
\text { open sea side } \\
\text { of Hatakijima } \\
\text { Surface }\end{array}$} & 96.0 & 5.0 & 94.5 & 0.5 & 1.5 & 98.5 & 0 & 1.0 & 99.0 & \\
\hline & 94.0 & 6.0 & 94.0 & 0 & 0.5 & 99.5 & 0 & 0.5 & 99.5 & 0 \\
\hline & 98.5 & 2.0 & 98.0 & 0 & 1.0 & 99.0 & 0 & 0.5 & 99.5 & \\
\hline \multirow{4}{*}{$\begin{array}{l}\text { Water from } \\
\text { land side of } \\
\text { Hatakejima } \\
\text { Surface }\end{array}$} & 88.5 & 13.5 & 86.5 & 0 & 7.5 & 92.5 & 0 & 73.0 & $27 \cdot 0$ & \\
\hline & 91.0 & 9.5 & 90.5 & 0 & 4.5 & 95.5 & 0 & 68.5 & 31.5 & 5 \\
\hline & 90.5 & 10.0 & 90.0 & 0 & 6.5 & 93.5 & 0 & 71.0 & 29.0 & \\
\hline & 91.5 & 9.5 & 90.5 & 0 & 6.5 & 93.5 & 0 & 3.0 & 97.0 & \\
\hline \multirow[t]{2}{*}{ Bottom (7) } & 90.0 & 10.0 & 84.0 & 6.0 & 4.5 & 95.5 & 0 & 2.5 & 97.5 & 2 \\
\hline & 94.0 & 7.0 & 93.0 & 0 & 4.0 & 96.0 & 0 & 3.0 & 97.0 & \\
\hline \multirow{3}{*}{$\begin{array}{l}\text { Sea water from } \\
\text { Tsunashirazu } \\
\text { cove } \\
\text { Surface }\end{array}$} & 96.0 & 5.0 & 95.0 & 0 & 3.5 & 96.5 & 0 & 3.5 & 96.5 & \\
\hline & 93.0 & 8.0 & 92.0 & 0 & 5.0 & 95.0 & 0 & 2.0 & 98.0 & 2 \\
\hline & 91.5 & 10.0 & 90.0 & 0 & 6.0 & 94.0 & 0 & 3.0 & 97.0 & \\
\hline & 94.0 & 6.5 & 93.5 & 0 & 5.5 & 94.5 & 0 & 6.0 & 94.0 & \\
\hline \multirow[t]{2}{*}{ Bottom (5) } & 94.0 & 7.0 & 93.0 & 0 & 4.0 & 96.0 & 0 & 4.5 & 95.5 & 2 \\
\hline & 92.0 & 9.0 & 91.0 & 0 & 6.0 & 94.0 & 0 & 3.5 & 96.5 & \\
\hline
\end{tabular}


Table 7. Results of the July 18. 1985 experiment with eggs of Anthocidaris crassispina.

Wind; 0, Test water temperature; $27^{\circ} \mathrm{C}, 5$ mins. old sperms, 3 hrs. old eggs. Ranking III 1985.

\begin{tabular}{|c|c|c|c|c|c|c|c|c|c|c|}
\hline \multirow{3}{*}{$\begin{array}{l}\text { Location } \\
\qquad \\
\text { (depth) }\end{array}$} & \multicolumn{9}{|c|}{ Time after insemination } & \multirow{3}{*}{$\begin{array}{l}\text { Degree of } \\
\text { inhibitory } \\
\text { effect }\end{array}$} \\
\hline & \multirow{2}{*}{ 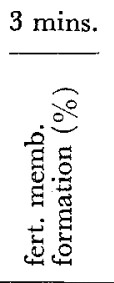 } & \multicolumn{3}{|c|}{60 mins. } & \multicolumn{3}{|c|}{16 hrs. } & \multicolumn{2}{|c|}{27 hrs. } & \\
\hline & & 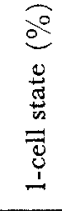 & 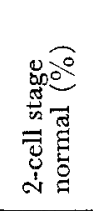 & 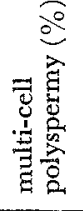 & 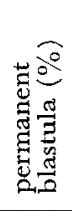 & 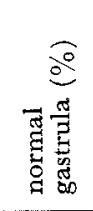 & $\frac{2}{2}$ & 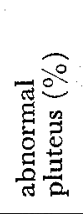 & 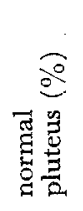 & \\
\hline \multicolumn{11}{|l|}{$(\mathrm{m})$} \\
\hline \multirow{3}{*}{$\begin{array}{l}\text { Running } \\
\text { sea water of } \\
\text { Laboratory }\end{array}$} & 98.0 & 1.5 & 98.5 & 0 & 0.5 & 99.5 & 0 & 1.0 & 99.0 & \\
\hline & 98.0 & 2.0 & 98.0 & 0 & 0.5 & 99.5 & 0 & 0.5 & 99.5 & 0 \\
\hline & 99.0 & 2.0 & 98.0 & 0 & 0 & 100 & 0 & 1.0 & 99.0 & \\
\hline \multirow{4}{*}{$\begin{array}{l}\text { Water from } \\
\text { open sea side } \\
\text { of Hatakejima } \\
\text { Surface }\end{array}$} & 98.0 & 2.0 & 98.0 & 0 & 0 & 100 & 0 & 0.5 & 99.5 & \\
\hline & 99.0 & 1.5 & 98.5 & 0 & 0.5 & 99.5 & 0 & 0.5 & 99.5 & 0 \\
\hline & 99.5 & 1.0 & 99.0 & 0 & 0.5 & 99.5 & 0 & 0.5 & 99.5 & \\
\hline & 97.5 & 3.0 & 97.0 & 0 & 1.0 & 99.0 & 0 & 2.5 & 97.5 & \\
\hline \multirow[t]{2}{*}{ Bottom (15) } & 98.0 & 2.5 & 97.5 & 0 & 0.5 & 99.5 & 0 & 3.0 & 97.0 & 0 \\
\hline & 97.0 & 3.5 & 95.5 & 1.0 & 0.5 & 99.5 & 0 & 2.0 & 98.0 & \\
\hline \multirow{4}{*}{$\begin{array}{l}\text { Water from } \\
\text { land side of } \\
\text { Hatakejima } \\
\text { Surface }\end{array}$} & 98.0 & 2.5 & 97.5 & 0 & 0.5 & 99.5 & 0 & 3.5 & 96.5 & \\
\hline & 98.5 & 3.0 & 96.0 & 1.0 & 1.0 & 99.0 & 0 & 5.5 & 94.5 & 1 \\
\hline & 97.0 & 3.5 & 95.0 & 1.5 & 2.0 & 98.0 & 0 & 3.0 & 97.0 & \\
\hline & 95.5 & 5.0 & 93.0 & 2.0 & 1.5 & 98.5 & 0 & 4.0 & 96.0 & \\
\hline \multirow[t]{2}{*}{ Bottom (7) } & 95.0 & 6.0 & 93.5 & 0.5 & 2.0 & 98.0 & 0 & 6.5 & 93.5 & 1 \\
\hline & 93.0 & 7.5 & 90.0 & 2.5 & 3.0 & 97.0 & 0 & 5.0 & 95.0 & \\
\hline \multirow{3}{*}{$\begin{array}{l}\text { Sea water from } \\
\text { Tsunashirazu } \\
\text { cove } \\
\text { Surface }\end{array}$} & 92.5 & 8.0 & 80.5 & 11.5 & 12.5 & 87.5 & 0 & 24.5 & 75.5 & \\
\hline & 94.0 & 6.5 & 80.5 & 13.0 & 11.0 & 89.0 & 0 & 19.5 & 80.5 & 4 \\
\hline & 93.0 & 7.5 & 78.5 & 14.0 & 9.5 & 90.5 & 0 & 21.0 & 79.0 & \\
\hline \multirow{3}{*}{ Bottom (5) } & 94.0 & 6.5 & 90.5 & 3.0 & 1.5 & 98.5 & 0 & 6.0 & 94.0 & \\
\hline & 95.0 & 6.0 & 92.0 & 2.0 & 2.5 & 97.5 & 0 & 7.0 & 93.0 & 1 \\
\hline & 95.5 & 5.0 & 93.5 & 1.5 & 2.0 & 98.0 & 0 & 8.5 & 91.5 & \\
\hline
\end{tabular}


Table 8. Results of the Aug. 28, 1985 experiment with eggs of Anthocidaris crassispina.

Wind; 0, Test water temperature; $27^{\circ} \mathrm{C}, 5$ mins. old sperms, 3 hrs. old eggs. Ranking III 1985.

\begin{tabular}{|c|c|c|c|c|c|c|c|c|c|c|}
\hline \multirow{3}{*}{$\begin{array}{l}\text { Location } \\
\qquad \text { (depth) }\end{array}$} & \multicolumn{9}{|c|}{ Time after insemination } & \multirow{3}{*}{$\begin{array}{l}\text { Degree of } \\
\text { inhibitory } \\
\text { effect }\end{array}$} \\
\hline & \multirow{2}{*}{$\frac{3 \text { mins. }}{200}$} & \multicolumn{3}{|c|}{60 mins. } & \multicolumn{3}{|c|}{15 hrs. } & \multicolumn{2}{|c|}{$24 \mathrm{hrs}}$. & \\
\hline & & 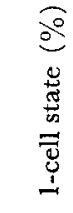 & 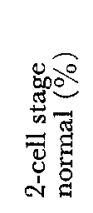 & 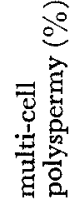 & 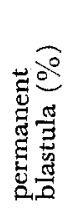 & 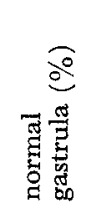 & 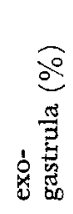 & 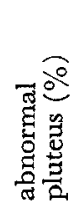 & 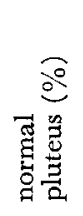 & \\
\hline \multicolumn{11}{|l|}{$(\mathrm{m})$} \\
\hline \multirow{3}{*}{$\begin{array}{l}\text { Running } \\
\text { sea water of } \\
\text { Laboratory }\end{array}$} & 99.0 & 1.5 & 98.5 & 0 & 1.0 & 99.0 & 0 & 0.5 & 99.5 & \\
\hline & 99.5 & 1.0 & 99.0 & 0 & 0.5 & 99.5 & 0 & 1.0 & 99.0 & 0 \\
\hline & 99.0 & 1.0 & 99.0 & 0 & 1.0 & 99.0 & 0 & 1.0 & 99.0 & \\
\hline \multirow{4}{*}{$\begin{array}{l}\text { Water from } \\
\text { open sea side } \\
\text { of Hatakejima } \\
\text { Surface }\end{array}$} & 99.5 & 1.0 & 99.0 & 0 & 0 & 100 & 0 & 1.0 & 99.0 & \\
\hline & 99.5 & 0.5 & 99.5 & 0 & 0.5 & 99.5 & 0 & 0.5 & 99.5 & 0 \\
\hline & 98.5 & 2.0 & 98.0 & 0 & 1.5 & 98.5 & 0 & 1.5 & 98.5 & \\
\hline & 99.0 & 1.0 & 99.0 & 0 & 0.5 & 99.5 & 0 & 0.5 & 99.5 & \\
\hline \multirow[t]{2}{*}{ Bottom (15) } & 98.0 & 2.5 & 97.5 & 0 & 1.0 & 99.0 & 0 & 1.0 & 99.0 & 0 \\
\hline & 98.0 & 2.0 & 98.0 & 0 & 1.5 & 98.5 & 0 & 2.0 & 98.0 & \\
\hline \multirow{4}{*}{$\begin{array}{l}\text { Water from } \\
\text { land side of } \\
\text { Hatakejima } \\
\text { Surface }\end{array}$} & 98.0 & 2.5 & 97.5 & 0 & 1.0 & 99.0 & 0 & 1.5 & 98.5 & \\
\hline & 96.5 & 4.5 & 95.5 & 0 & 1.5 & 98.5 & 0 & 2.5 & 97.5 & 0 \\
\hline & 97.0 & 3.5 & 96.5 & 0 & 2.0 & 98.0 & 0 & 2.0 & 98.0 & \\
\hline & 97.0 & 3.5 & 96.5 & 0 & 1.5 & 98.5 & 0 & 2.5 & 97.5 & \\
\hline \multirow[t]{2}{*}{ Bottom (7) } & 94.5 & 7.0 & 93.0 & 0 & 2.0 & 98.0 & 0 & 3.5 & 96.5 & 0 \\
\hline & 96.0 & 4.5 & 95.5 & 0 & 3.0 & 97.0 & 0 & 3.0 & 97.0 & \\
\hline \multirow{3}{*}{$\begin{array}{l}\text { Sea water from } \\
\text { Tsunashirazu } \\
\text { cove } \\
\text { Surface }\end{array}$} & 56.5 & 45.5 & 54.5 & 0 & 6.0 & 94.0 & 0 & 5.0 & 95.0 & \\
\hline & 63.5 & 39.0 & 61.0 & 0 & 4.5 & 95.5 & 0 & 4.5 & 95.5 & 4 \\
\hline & 58.0 & 43.0 & 57.0 & 0 & 8.5 & 91.5 & 0 & 7.5 & 92.5 & \\
\hline \multirow{3}{*}{ Bottom (5) } & 81.5 & 19.5 & 77.0 & 3.5 & 1.0 & 99.0 & 0 & 2.5 & 97.5 & \\
\hline & 78.0 & 24.0 & 75.0 & 1.0 & 3.5 & 96.5 & 0 & 3.5 & 96.5 & 2 \\
\hline & 75.5 & 25.5 & 72.5 & 2.0 & 4.0 & 96.0 & 0 & 4.0 & 96.0 & \\
\hline
\end{tabular}


Table 9. Results of the Jan. 23, 1986 experiment with eggs of Hemicentrotus pulcherrimus.

Wind; NW1, Test water temperature; $19^{\circ} \mathrm{C}, 5$ mins. old sperms, 6 hrs. old eggs. Ranking III 1985.

\begin{tabular}{|c|c|c|c|c|c|c|c|c|c|c|}
\hline \multirow{3}{*}{ Location } & \multicolumn{9}{|c|}{ Time after insemination } & \multirow{3}{*}{$\begin{array}{l}\text { Degree of } \\
\text { inhibitory } \\
\text { effect }\end{array}$} \\
\hline & \multirow{2}{*}{$\frac{3 \text { mins. }}{\text { O̊ }}$} & \multicolumn{3}{|c|}{65 mins. } & \multicolumn{3}{|c|}{24 hrs. } & \multicolumn{2}{|c|}{48 hrs. } & \\
\hline & & 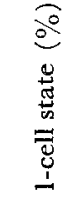 & 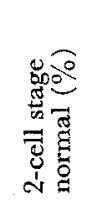 & 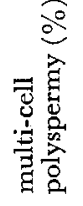 & 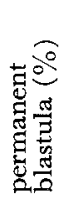 & 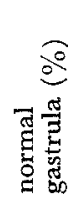 & 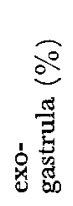 & 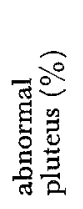 & 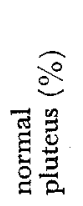 & \\
\hline \multicolumn{11}{|l|}{$(\mathrm{m})$} \\
\hline \multirow{3}{*}{$\begin{array}{l}\text { Running } \\
\text { sea water of } \\
\text { Laboratory }\end{array}$} & 99.0 & 1.5 & 98.5 & 0 & 0.5 & 99.5 & 0 & 1.0 & 99.0 & \\
\hline & 100 & 0.5 & 99.5 & 0 & 1.0 & 99.0 & 0 & 0.5 & 99.5 & 0 \\
\hline & 99.5 & 2.0 & 98.0 & 0 & 1.5 & 98.5 & 0 & 1.0 & 99.0 & \\
\hline \multirow{4}{*}{$\begin{array}{l}\text { Water from } \\
\text { land side of } \\
\text { Hatakejima } \\
\text { Surface }\end{array}$} & 93.0 & 8.0 & 92.0 & 0 & 4.5 & 95.5 & 0 & 2.5 & 97.5 & \\
\hline & 99.5 & 1.0 & 99.0 & 0 & 3.5 & 96.5 & 0 & 1.5 & 98.5 & 1 \\
\hline & 92.0 & 9.5 & 90.5 & 0 & 2.5 & 97.5 & 0 & 1.0 & 99.0 & \\
\hline & 85.5 & 18.5 & 81.5 & 0 & 11.0 & 89.0 & 0 & 18.5 & 81.5 & \\
\hline \multirow[t]{2}{*}{ Bottom (7) } & 91.0 & 9.5 & 90.5 & 0 & 7.5 & 92.5 & 0 & 27.5 & 72.5 & 5 \\
\hline & 88.5 & 14.0 & 86.0 & 0 & 18.0 & 82.0 & 0 & 23.0 & 77.0 & \\
\hline \multirow{3}{*}{$\begin{array}{l}\text { Sea water from } \\
\text { Tsunashirazu } \\
\text { cove } \\
\text { Surface }\end{array}$} & 90.5 & 10.5 & 89.5 & 0 & 6.0 & 94.0 & 0 & 8.5 & 91.5 & \\
\hline & 94.0 & 8.0 & 92.0 & 0 & 3.5 & 96.5 & 0 & 13.0 & 87.0 & 2 \\
\hline & 91.5 & 10.0 & 90.0 & 0 & 7.5 & 92.5 & 0 & 7.0 & 93.0 & \\
\hline \multirow{3}{*}{ Bottom (5) } & 96.0 & 5.0 & 95.0 & 0 & 7.0 & 93.0 & 0 & 16.0 & 84.0 & \\
\hline & 97.5 & 3.0 & 97.0 & 0 & 4.0 & 96.0 & 0 & 15.5 & 84.5 & 3 \\
\hline & 90.0 & 11.5 & 88.5 & 0 & 8.5 & 91.5 & 0 & 9.5 & $90 \cdot 5$ & \\
\hline
\end{tabular}


Table 10. Results of the Mar. 28, 1986 experiment with eggs of Hemicentrotus pulcherrimus. Wind; 0, Test water temperature; $19^{\circ} \mathrm{C}, 5$ mins. old sperms, 6 hrs. old eggs. Ranking III 1985.

\begin{tabular}{|c|c|c|c|c|c|c|c|c|c|c|}
\hline \multirow{3}{*}{ Location } & \multicolumn{9}{|c|}{ Time after insemination } & \multirow{3}{*}{$\begin{array}{l}\text { Degree of } \\
\text { inhibitory } \\
\text { effect }\end{array}$} \\
\hline & \multirow{2}{*}{\begin{tabular}{l}
3 mins. \\
\multirow{2}{0}{} \\
\end{tabular}} & \multicolumn{3}{|c|}{65 mins. } & \multicolumn{3}{|c|}{$24 \mathrm{hrs}$. } & \multicolumn{2}{|c|}{$48 \mathrm{hrs.}$} & \\
\hline & & 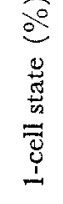 & 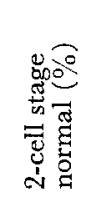 & 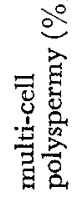 & 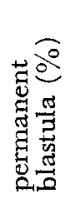 & 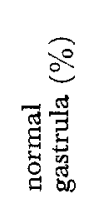 & 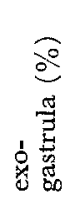 & 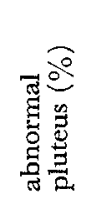 & 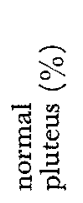 & \\
\hline \multicolumn{11}{|l|}{$(\mathrm{m})$} \\
\hline \multirow{3}{*}{$\begin{array}{l}\text { Running } \\
\text { sea water of } \\
\text { Laboratory }\end{array}$} & 96.5 & 4.5 & 95.5 & 0 & 1.5 & 98.5 & 0 & 0.5 & 99.5 & \\
\hline & 97.5 & 4.0 & 96.0 & 0 & 1.0 & 99.0 & 0 & 1.5 & 98.5 & 0 \\
\hline & 98.0 & 2.5 & 97.5 & 0 & 0.5 & 99.5 & 0 & 1.5 & 98.5 & \\
\hline \multirow{3}{*}{$\begin{array}{l}\text { Water from } \\
\text { open sea side } \\
\text { of Hatakejima } \\
\text { Surface }\end{array}$} & 96.5 & 4.5 & 95.5 & 0 & 1.0 & 99.0 & 0 & 76.5 & 23.5 & \\
\hline & 97.0 & 4.0 & 96.0 & 0 & 1.0 & 99.0 & 0 & 100 & 0 & 5 \\
\hline & 98.5 & 2.0 & 98.0 & 0 & 0.5 & 99.5 & 0 & 100 & 0 & \\
\hline \multirow{4}{*}{$\begin{array}{l}\text { Water from } \\
\text { land side of } \\
\text { Hatakejima } \\
\text { Surface }\end{array}$} & 96.5 & 4.5 & 95.5 & 0 & 3.5 & $96 \cdot 5$ & 0 & 16.5 & 83.5 & \\
\hline & 96.5 & 5.0 & 95.0 & 0 & 2.5 & 97.5 & 0 & 100 & 0 & 5 \\
\hline & 97.5 & 3.0 & 97.0 & 0 & 2.0 & 98.0 & 0 & 100 & 0 & \\
\hline & 96.0 & 5.5 & 94.5 & 0 & 2.5 & 97.5 & 0 & 7.0 & 93.0 & \\
\hline \multirow[t]{2}{*}{ Bottom (7) } & 94.0 & 6.0 & 94.0 & 0 & 2.0 & 98.0 & 0 & 25.5 & 74.5 & 5 \\
\hline & 97.5 & 3.0 & 97.0 & 0 & 1.5 & 98.5 & 0 & 19.0 & 81.0 & \\
\hline \multirow{3}{*}{$\begin{array}{l}\text { Sea water from } \\
\text { Tsunashirazu } \\
\text { cove } \\
\text { Surface }\end{array}$} & 96.0 & 5.0 & 95.0 & 0 & 4.5 & 95.5 & 0 & 26.5 & 73.5 & \\
\hline & 95.0 & 5.5 & 94.5 & 0 & 5.0 & 95.0 & 0 & 53.5 & 46.5 & 5 \\
\hline & 98.5 & 3.0 & 97.0 & 0 & 3.0 & 97.0 & 0 & 63.5 & 36.5 & \\
\hline \multirow{3}{*}{ Bottom (5) } & 95.5 & 5.5 & 94.5 & 0 & 6.5 & 93.5 & 0 & 3.5 & 96.5 & \\
\hline & 96.6 & 5.0 & 95.0 & 0 & 5.5 & 94.5 & 0 & 4.0 & 96.0 & 1 \\
\hline & 97.0 & 3.0 & 97.0 & 0 & 3.5 & 96.5 & 0 & 3.0 & 97.0 & \\
\hline
\end{tabular}


Table 11. Results of the Sep. 4, 1986 experiment with eggs of Anthocidaris crassispina.

Wind; 0, Test water temperature; $27^{\circ} \mathrm{C}, 5$ mins. old sperms, 3 hrs. old eggs. Ranking III 1985.

\begin{tabular}{|c|c|c|c|c|c|c|c|c|c|c|}
\hline \multirow{3}{*}{$\begin{array}{l}\text { Location } \\
\qquad \text { (depth) }\end{array}$} & \multicolumn{9}{|c|}{ Time after insemination } & \multirow{3}{*}{$\begin{array}{l}\text { Degree of } \\
\text { inhibitory } \\
\text { effect }\end{array}$} \\
\hline & \multirow{2}{*}{3 mins. } & \multicolumn{3}{|c|}{60 mins. } & \multicolumn{3}{|c|}{$18 \mathrm{hrs}}$. & \multicolumn{2}{|c|}{30 hrs. } & \\
\hline & & 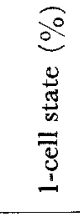 & 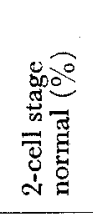 & 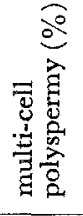 & 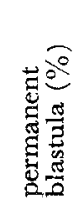 & 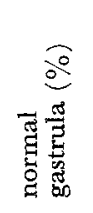 & $\frac{2}{2}$ & 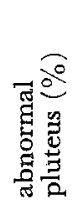 & 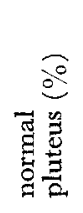 & \\
\hline \multicolumn{11}{|l|}{$(\mathrm{m})$} \\
\hline \multirow{3}{*}{$\begin{array}{l}\text { Running } \\
\text { sea water of } \\
\text { Laboratory }\end{array}$} & 99.5 & 0.5 & 99.5 & 0 & 0.5 & 99.5 & 0 & 0.5 & 99.5 & \\
\hline & 99.0 & 1.0 & 99.0 & 0 & 0.5 & 99.5 & 0 & $1.0^{\circ}$ & 99.0 & 0 \\
\hline & 98.0 & 2.5 & 97.5 & 0 & 1.0 & 99.0 & 0 & 0.5 & 99.5 & \\
\hline \multirow{4}{*}{$\begin{array}{l}\text { Water from } \\
\text { open sea side } \\
\text { of Hatakejima } \\
\text { Surface }\end{array}$} & 99.0 & 1.0 & 98.0 & 1.0 & 1.0 & 99.0 & 0 & 2.0 & 98.0 & \\
\hline & 98.5 & 1.5 & 97.0 & 1.5 & 1.5 & 98.5 & 0 & 1.5 & 98.5 & 0 \\
\hline & 98.0 & 1.5 & 97.0 & 1.5 & 1.5 & 98.5 & 0 & 0.5 & 99.5 & \\
\hline & 98.5 & 1.0 & 98.5 & 0.5 & 2.0 & 98.0 & 0 & 3.0 & 97.0 & \\
\hline \multirow[t]{2}{*}{ Bottom (15) } & 98.0 & 2.0 & 97.0 & 1.0 & 2.0 & 98.0 & 0 & 2.5 & 97.5 & 0 \\
\hline & 97.0 & 3.0 & 96.0 & 1.0 & 1.5 & 98.5 & 0 & 1.5 & 98.5 & \\
\hline \multirow{4}{*}{$\begin{array}{l}\text { Water from } \\
\text { land side of } \\
\text { Hatakejima } \\
\text { Surface }\end{array}$} & 97.0 & 3.0 & 95.5 & 1.5 & 3.5 & 96.5 & 0 & 3.5 & 96.5 & \\
\hline & 96.0 & 4.0 & 94.0 & 2.0 & 5.0 & 95.0 & 0 & 2.5 & 97.5 & 1 \\
\hline & 96.0 & 4.0 & 95.0 & 1.0 & 4.5 & 95.5 & 0 & 3.0 & 97.0 & \\
\hline & 96.0 & 3.0 & 93.0 & 4.0 & 4.5 & 95.5 & 0 & 4.5 & 95.5 & \\
\hline \multirow[t]{2}{*}{ Bottom (7) } & 93.0 & 7.0 & 91.0 & 2.0 & 5.0 & 95.0 & 0 & 3.0 & 97.0 & 1 \\
\hline & 94.0 & 6.0 & 93.0 & 1.0 & 4.5 & 95.5 & 0 & 2.5 & 97.5 & \\
\hline \multirow{3}{*}{$\begin{array}{l}\text { Sea water from } \\
\text { Tsunashirazu } \\
\text { cove } \\
\text { Surface }\end{array}$} & 18.5 & 84.0 & 16.0 & 0 & 100 & 0 & 0 & & & \\
\hline & 15.0 & 86.0 & 14.0 & 0 & 100 & 0 & 0 & & & 5 \\
\hline & 26.0 & 75.0 & 25.0 & 0 & 100 & 0 & 0 & & & \\
\hline \multirow{3}{*}{ Bottom (5) } & 81.5 & 21.0 & 74.5 & 4.5 & 4.0 & 96.0 & 0 & 5.0 & 95.0 & \\
\hline & 80.0 & 20.0 & 78.0 & 2.0 & 4.5 & 95.5 & 0 & 4.5 & 95.5 & 2 \\
\hline & 77.5 & 21.0 & 76.0 & 3.0 & 4.5 & 95.5 & 0 & 6.0 & 94.0 & \\
\hline
\end{tabular}


Table 12. Results of the Jan. 17, 1987 experiment with eggs of Hemicentrolus pulcherrimus. Wind; 0, Test water temperature; $19^{\circ} \mathrm{G}, 5$ mins. old sperms, 6 hrs. old eggs. Ranking III 1985.

\begin{tabular}{|c|c|c|c|c|c|c|c|c|c|c|}
\hline \multirow{3}{*}{$\begin{array}{l}\text { Location } \\
\qquad \text { (depth) }\end{array}$} & \multicolumn{9}{|c|}{ Time after insemination } & \multirow{3}{*}{$\begin{array}{l}\text { Degree of } \\
\text { inhibitory } \\
\text { effect }\end{array}$} \\
\hline & \multirow{2}{*}{3 mins. } & \multicolumn{3}{|c|}{75 mins. } & \multicolumn{3}{|c|}{22 hrs. } & \multicolumn{2}{|c|}{45 hrs. } & \\
\hline & & 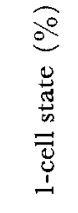 & 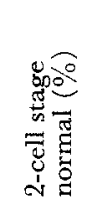 & 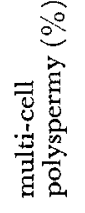 & 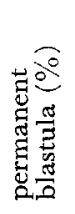 & 总 & 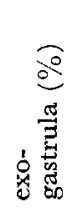 & 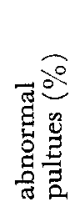 & 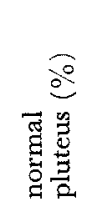 & \\
\hline \multicolumn{11}{|l|}{$(\mathrm{m})$} \\
\hline \multirow{3}{*}{$\begin{array}{l}\text { Running } \\
\text { sea water of } \\
\text { Laboratory }\end{array}$} & 98.0 & 3.0 & 97.0 & 0 & 0 & 100 & 0 & 1.0 & 99.0 & \\
\hline & 99.5 & 1.0 & 99.0 & 0 & 1.0 & 99.0 & 0 & 0.5 & 99.5 & 1 \\
\hline & 83.0 & 18.0 & 82.0 & 0 & 1.5 & 98.5 & 0 & 0.5 & 99.5 & \\
\hline \multirow{3}{*}{$\begin{array}{l}\text { Water from } \\
\text { open sea side } \\
\text { of Hatakejima } \\
\text { Surface }\end{array}$} & 98.5 & 1.5 & 98.0 & 0.5 & 0.5 & 99.5 & 0 & 0 & 100 & \\
\hline & 99.5 & 1.0 & 99.0 & 0 & 1.0 & 99.0 & 0 & 0.5 & 99.5 & 1 \\
\hline & 87.5 & 12.5 & 87.0 & 0.5 & 1.5 & 98.5 & 0 & 1.0 & 99.0 & \\
\hline \multirow{4}{*}{$\begin{array}{l}\text { Water from } \\
\text { land side of } \\
\text { Hatakejima. } \\
\text { Surface }\end{array}$} & 98.0 & 2.5 & 97.0 & 0.5 & 3.5 & 96.5 & 0 & 1.0 & 99.0 & \\
\hline & 99.0 & 2.0 & 98.0 & 0 & 3.0 & 97.0 & 0 & 1.5 & 98.5 & 1 \\
\hline & 83.5 & 17.0 & 82.5 & 0.5 & 5.0 & 95.0 & 0 & 1.0 & 99.0 & \\
\hline & 81.0 & 20.0 & 79.0 & 1.0 & 11.0 & 89.0 & 0 & 2.0 & 98.0 & \\
\hline \multirow[t]{2}{*}{ Bottom (7) } & 83.5 & 17.0 & 82.0 & 1.0 & 10.5 & 89.5 & 0 & 2.5 & 97.5 & 3 \\
\hline & 73.0 & 28.0 & 70.0 & 2.0 & 16.5 & 83.5 & 0 & 1.5 & 98.5 & \\
\hline \multirow{3}{*}{$\begin{array}{l}\text { Sea water from } \\
\text { Tsunashirazu } \\
\text { cove } \\
\text { Surface }\end{array}$} & 85.0 & 32.5 & 65.0 & 2.5 & 19.5 & 80.5 & 0 & 2.0 & 98.0 & \\
\hline & 87.5 & 35.0 & 61.0 & 4.0 & 19.0 & 81.0 & 0 & 2.5 & 97.5 & 4 \\
\hline & 79.5 & 31.0 & 66.0 & 3.0 & 22.0 & 78.0 & 0 & 3.0 & 97.0 & \\
\hline \multirow{3}{*}{ Bottom (5) } & 62.0 & 39.0 & 59.0 & 2.0 & 21.5 & 78.5 & 0 & 2.0 & 98.0 & \\
\hline & 78.0 & 40.5 & 54.5 & 5.0 & 24.0 & 76.0 & 0 & 3.0 & 97.0 & 5 \\
\hline & 75.0 & 36.0 & 60.0 & 4.0 & 29.0 & 71.0 & 0 & 2.5 & 97.5 & \\
\hline
\end{tabular}


Table 13. Results of the Mar. 19, 1987 experiment with eggs of Hemicentrotus pulcherrimus. Wind; 0., Test water temperature; $19^{\circ} \mathrm{C}, 5$ mins. old sperms, 6 hrs. old eggs. Ranking III 1985.

\begin{tabular}{|c|c|c|c|c|c|c|c|c|c|c|}
\hline \multirow{3}{*}{ Location } & \multicolumn{9}{|c|}{ Time after insemination } & \multirow{3}{*}{$\begin{array}{l}\text { Degree of } \\
\text { inhibitory } \\
\text { effect }\end{array}$} \\
\hline & \multirow{2}{*}{$\frac{3 \text { mins. }}{\text { क् }}$} & \multicolumn{3}{|c|}{75 mins. } & \multicolumn{3}{|c|}{24 hrs. } & \multicolumn{2}{|c|}{48 hrs. } & \\
\hline & & 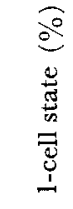 & 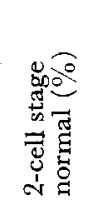 & 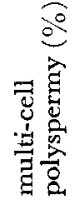 & 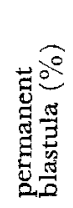 & 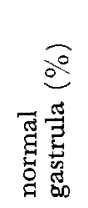 & 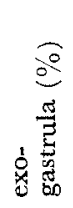 & 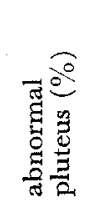 & 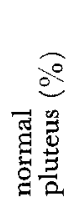 & \\
\hline \multicolumn{11}{|l|}{$(\mathrm{m})$} \\
\hline \multirow{3}{*}{$\begin{array}{l}\text { Running } \\
\text { sea water of } \\
\text { Laboratory }\end{array}$} & 98.5 & 2.5 & 97.5 & 0 & 1.0 & 99.0 & 0 & 1.0 & 99.0 & \\
\hline & 99.0 & 1.5 & 98.5 & 0 & 0.5 & 99.5 & 0 & 1.5 & 98.5 & 0 \\
\hline & 98.5 & 2.0 & 97.0 & 1.0 & 1.5 & 98.5 & 0 & 0.5 & 99.5 & \\
\hline \multirow{3}{*}{$\begin{array}{l}\text { Water from } \\
\text { open sea side } \\
\text { of Hatakejima } \\
\text { Surface }\end{array}$} & 99.0 & 1.5 & 98.5 & 0 & 1.0 & 99.0 & 0 & 1.0 & 99.0 & \\
\hline & 99.5 & 1.0 & 99.0 & 0 & 0.5 & 99.5 & 0 & 1.0 & 99.0 & 0 \\
\hline & 98.5 & 1.5 & 97.0 & 1.5 & 2.0 & 98.0 & 0 & 1.5 & 98.5 & \\
\hline \multirow{4}{*}{$\begin{array}{l}\text { Water from } \\
\text { land side of } \\
\text { Hatakejima } \\
\text { Surface }\end{array}$} & 96.0 & 4.0 & 95.0 & 1.0 & 2.0 & 98.0 & 0 & 1.5 & 98.5 & \\
\hline & 97.0 & 3.5 & 92.0 & 4.5 & 2.5 & 97.5 & 0 & 1.5 & 98.5 & 1 \\
\hline & 96.5 & 7.5 & 89.0 & 3.5 & 3.0 & 97.0 & 0 & 2.0 & 98.0 & \\
\hline & 97.0 & 10.0 & 72.5 & 17.5 & 8.5 & 91.5 & 0 & 100 & 0 & \\
\hline \multirow[t]{2}{*}{ Bottom (7) } & 98.0 & 8.0 & 71.0 & 21.0 & 10.0 & 90.0 & 0 & 65.0 & 35.0 & 5 \\
\hline & 97.0 & 11.0 & 65.5 & 23.5 & 13.0 & 87.0 & 0 & 100 & 0 & \\
\hline \multirow{3}{*}{$\begin{array}{l}\text { Sea water from } \\
\text { Tsunashirazu } \\
\text { cove } \\
\text { Surface }\end{array}$} & 8.0 & 93.0 & 7.0 & 0 & & & & & & \\
\hline & 7.0 & 93.5 & 6.5 & 0 & & & & & & 5 \\
\hline & 5.5 & 95.0 & 5.0 & 0 & & & & & & \\
\hline \multirow{3}{*}{ Bottom (5) } & 94.0 & 15.0 & 61.5 & 23.5 & 13.5 & 86.5 & 0 & 100 & 0 & \\
\hline & 95.0 & 7.5 & 64.5 & 28.0 & 16.5 & 83.5 & 0 & 100 & 0 & 5 \\
\hline & 93.0 & 11.5 & 57.5 & 31.0 & 83.0 & 17.0 & 0 & 100 & 0 & \\
\hline
\end{tabular}


Table 14. Results of the June 10, 1987 experiment with eggs of Anthocidaris crassispina. Wind; 0, Test water temperature; $25^{\circ} \mathrm{C}, 5$ mins. old sperms, 3 hrs. old eggs. Ranking III 1985.

\begin{tabular}{|c|c|c|c|c|c|c|c|c|c|c|}
\hline \multirow{3}{*}{$\begin{array}{l}\text { Location } \\
\qquad \begin{array}{l}\text { (depth) }\end{array}\end{array}$} & \multicolumn{9}{|c|}{ Time after insemination } & \multirow{3}{*}{$\begin{array}{l}\text { Degree of } \\
\text { inhibitory } \\
\text { effect }\end{array}$} \\
\hline & \multirow{2}{*}{$\frac{3 \text { mins. }}{\text { (20 }}$} & \multicolumn{3}{|c|}{70 mins. } & \multicolumn{3}{|c|}{15 hrs. } & \multicolumn{2}{|c|}{$24 \mathrm{hrs}$. } & \\
\hline & & 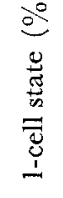 & 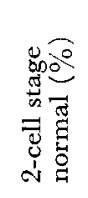 & 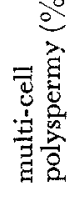 & 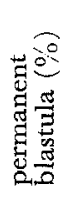 & 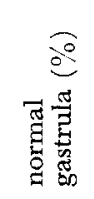 & 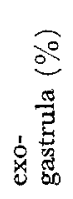 & 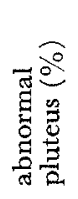 & 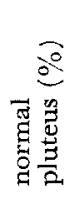 & \\
\hline \multicolumn{11}{|l|}{$(\mathrm{m})$} \\
\hline \multirow{3}{*}{$\begin{array}{l}\text { Running } \\
\text { sea water of } \\
\text { Laboratory }\end{array}$} & 99.5 & 1.0 & 99.0 & 0 & 0.5 & 99.5 & 0 & 1.0 & 99.0 & \\
\hline & 99.0 & 1.0 & 99.0 & 0 & 0 & 100 & 0 & 2.0 & 98.0 & 0 \\
\hline & 98.0 & 2.5 & 97.5 & 0 & 1.0 & 99.0 & 0 & 1.5 & 98.5 & \\
\hline \multirow{4}{*}{$\begin{array}{l}\text { Water from } \\
\text { open sea side } \\
\text { of Hatakejima } \\
\text { Surface }\end{array}$} & 99.5 & 1.0 & 99.0 & 0 & 1.0 & 99.0 & 0 & 1.5 & 98.5 & \\
\hline & 99.5 & 0.5 & 99.5 & 0 & 0.5 & 99.5 & 0 & 1.5 & 98.5 & 0 \\
\hline & 98.5 & 2.0 & 98.0 & 0 & 0.5 & 99.5 & 0 & 2.0 & 98.0 & \\
\hline & 98.0 & 3.0 & 97.0 & 0 & 1.5 & 98.5 & 0 & 2.0 & 98.0 & \\
\hline \multirow[t]{2}{*}{ Bottom (15) } & 98.5 & 2.0 & 98.0 & 0 & 2.0 & 98.0 & 0 & 1.5 & 98.5 & 0 \\
\hline & 99.0 & 2.5 & 97.5 & 0 & 1.0 & 99.0 & 0 & 2.5 & 97.5 & \\
\hline \multirow{3}{*}{$\begin{array}{l}\text { Water from } \\
\text { Land side of } \\
\text { Hatakejima } \\
\text { Surface }\end{array}$} & 97.0 & 4.0 & 95.0 & 1.0 & 4.0 & 96.0 & 0 & 3.5 & 96.5 & \\
\hline & 98.5 & 2.0 & 97.5 & 0.5 & 3.5 & 96.5 & 0 & 4.5 & 95.5 & 1 \\
\hline & 97.5 & 2.5 & 97.0 & 0.5 & 4.5 & 95.5 & 0 & 5.0 & 95.0 & \\
\hline \multirow{3}{*}{ Bottom (7) } & 98.5 & 2.0 & 97.0 & 1.0 & 4.0 & 96.0 & 0 & 9.5 & 90.5 & \\
\hline & 98.0 & 2.5 & 96.0 & 1.5 & 5.0 & 95.0 & 0 & 9.0 & 91.0 & 2 \\
\hline & 96.5 & 4.0 & 94.0 & 2.0 & 3.5 & 96.5 & 0 & 9.5 & 90.5 & \\
\hline \multirow{3}{*}{$\begin{array}{l}\text { Sea water from } \\
\text { Tsunashirazu } \\
\text { cove } \\
\text { Surface }\end{array}$} & 98.0 & 3.0 & 96.0 & 1.0 & 4.0 & 96.0 & 0 & 4.5 & 95.5 & \\
\hline & 97.5 & 3.5 & 96.0 & 0.5 & 3.0 & 97.0 & 0 & 5.0 & 95.0 & 1 \\
\hline & 96.0 & 5.0 & 94.0 & 1.0 & 3.5 & 96.5 & 0 & 4.0 & 96.0 & \\
\hline \multirow{3}{*}{ Bottom (5) } & 95.0 & 5.5 & 92.5 & 2.0 & 4.5 & 95.5 & 0 & 10.5 & 89.5 & \\
\hline & 96.0 & 3.5 & 94.0 & 2.5 & 6.0 & 94.0 & 0 & 9.0 & 91.0 & 2 \\
\hline & 94.0 & 6.5 & 90.0 & 3.5 & 5.0 & 95.0 & 0 & 10.0 & 90.0 & \\
\hline
\end{tabular}


Table 15. Results of the Aug. 11, 1987 experiment with eggs of Anthocidaris crassispina.

Wind; 0, Test water temperature; $27^{\circ} \mathrm{C}, 5$ mins. old sperms, 3 hrs. old eggs. Ranking III 1985.

\begin{tabular}{|c|c|c|c|c|c|c|c|c|c|c|}
\hline \multirow{3}{*}{$\begin{array}{l}\text { Location } \\
\qquad \text { (depth) }\end{array}$} & \multicolumn{9}{|c|}{ Time after insemination } & \multirow{3}{*}{$\begin{array}{l}\text { Degree of } \\
\text { inhibitory } \\
\text { effect }\end{array}$} \\
\hline & \multirow{2}{*}{ 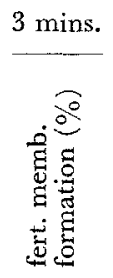 } & \multicolumn{3}{|c|}{60 mins. } & \multicolumn{3}{|c|}{$18 \mathrm{hrs}}$. & \multicolumn{2}{|c|}{$24 \mathrm{hrs}$. } & \\
\hline & & 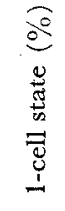 & 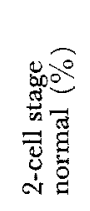 & 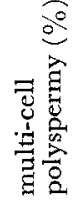 & 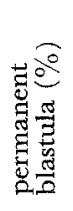 & 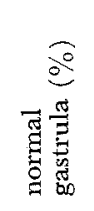 & 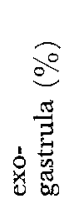 & 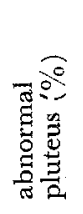 & 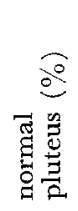 & \\
\hline \multicolumn{11}{|l|}{$(\mathrm{m})$} \\
\hline \multirow{3}{*}{$\begin{array}{l}\text { Running } \\
\text { sea water of } \\
\text { Laboratory }\end{array}$} & 93.5 & 7.0 & 93.0 & 0 & 0.5 & 99.5 & 0 & 0.5 & 99.5 & \\
\hline & 9.95 & 1.0 & 99.0 & 0 & 1.0 & 99.0 & 0 & 1.0 & 99.0 & 0 \\
\hline & 99.0 & 1.5 & 98.5 & 0 & 1.0 & 99.0 & 0 & 1.0 & 99.0 & \\
\hline \multirow{4}{*}{$\begin{array}{l}\text { Water from } \\
\text { open sea side } \\
\text { of Hatakejima } \\
\text { Surface }\end{array}$} & 94.5 & 5.5 & 94.5 & 0 & 1.0 & 99.0 & 0 & 0.5 & 99.5 & \\
\hline & 99.5 & 0.5 & 99.5 & 0 & 0.5 & 99.5 & 0 & 0.5 & 99.5 & 0 \\
\hline & 99.0 & 1.0 & 99.0 & 0 & 0.5 & 99.5 & 0 & 1.0 & 99.0 & \\
\hline & 94.5 & 6.0 & 94.0 & 0 & 0.5 & 99.5 & 0 & 1.0 & 99.0 & \\
\hline \multirow[t]{2}{*}{ Bottom (15) } & 98.5 & 2.0 & 98.0 & 0 & 1.0 & 99.0 & 0 & 1.5 & 98.5 & 0 \\
\hline & 98.0 & 2.5 & 97.5 & 0 & 1.0 & 99.0 & 0 & 2.0 & 98.0 & \\
\hline \multirow{4}{*}{$\begin{array}{l}\text { Water from } \\
\text { land side of } \\
\text { Hatakejima } \\
\text { Surface }\end{array}$} & 93.5 & 7.0 & 93.0 & 0 & 1.5 & 98.5 & 0 & 2.0 & 98.0 & \\
\hline & 98.0 & 2.5 & 97.5 & 0 & 2.0 & 98.0 & 0 & 5.0 & 95.0 & 1 \\
\hline & 97.5 & 3.0 & 97.0 & 0 & 1.5 & 98.5 & 0 & 5.5 & 94.5 & \\
\hline & 93.0 & 6.5 & 93.5 & 0 & 2.0 & 98.0 & 0 & 10.0 & 90.0 & \\
\hline \multirow[t]{2}{*}{ Bottom (7) } & 98.5 & 3.0 & 97.0 & 0 & 2.0 & 98.0 & 0 & 11.0 & 89.0 & 2 \\
\hline & 98.5 & 2.0 & 98.0 & 0 & 2.5 & 97.5 & 0 & 12.0 & 88.0 & \\
\hline \multirow{3}{*}{$\begin{array}{l}\text { Sea water from } \\
\text { Tsunashirazu } \\
\text { cove } \\
\text { Surface }\end{array}$} & 92.0 & 8.0 & 90.5 & 1.5 & 1.5 & 98.5 & 0 & 11.0 & 89.0 & \\
\hline & 96.5 & 4.0 & 94.0 & 2.0 & 2.5 & 97.5 & 0 & 13.0 & 87.0 & 2 \\
\hline & 97.0 & 4.5 & 92.5 & 3.0 & 3.0 & 97.0 & 0 & 10.5 & 89.5 & \\
\hline \multirow{3}{*}{ Bottom (5) } & 93.0 & 8.0 & 92.0 & 0 & 2.0 & 98.0 & 0 & 9.5 & 90.5 & \\
\hline & 95.0 & 6.0 & 94.0 & 0 & 3.0 & 97.0 & 0 & 12.5 & 87.5 & 2 \\
\hline & 95.5 & 5.0 & 95.0 & 0 & 3.0 & 97.0 & 0 & 14.0 & 86.0 & \\
\hline
\end{tabular}


Table 16. Results of the Mar. 29, 1988 experiment with eggs of Hemicentrotus pulcherrimus.

Wind; 0, Test water temperature; $19^{\circ} \mathrm{C}, 5$ mins. old sperms, 6 hrs.. old eggs. Ranking III 1985.

\begin{tabular}{|c|c|c|c|c|c|c|c|c|c|c|}
\hline \multirow{3}{*}{ Location } & \multicolumn{9}{|c|}{ Time after insemination } & \multirow{3}{*}{$\begin{array}{l}\text { Degree of } \\
\text { inhibitory } \\
\text { effect }\end{array}$} \\
\hline & \multirow{2}{*}{3 mins. } & \multicolumn{3}{|c|}{75 mins. } & \multicolumn{3}{|c|}{$24 \mathrm{hrs}}$. & \multicolumn{2}{|c|}{$48 \mathrm{hrs}$. } & \\
\hline & & 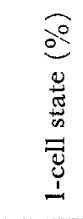 & 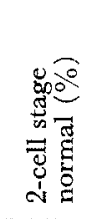 & 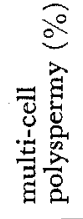 & 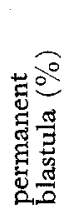 & 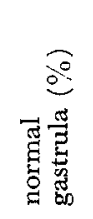 & 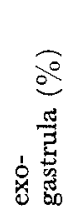 & 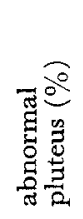 & 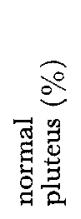 & \\
\hline \multicolumn{11}{|l|}{$(\mathrm{m})$} \\
\hline \multirow{3}{*}{$\begin{array}{l}\text { Running } \\
\text { sea water of } \\
\text { Laboratory }\end{array}$} & 100 & 1.0 & 99.0 & 0 & 0.5 & 99.5 & 0 & 0.5 & 99.5 & \\
\hline & 99.5 & 1.0 & 99.0 & 0 & 1.0 & 99.0 & 0 & 1.0 & 99.0 & 0 \\
\hline & 98.0 & 3.5 & 96.5 & 0 & 1.5 & 98.5 & 0 & 1.0 & 99.0 & \\
\hline \multirow{4}{*}{$\begin{array}{l}\text { Water from } \\
\text { open sea side } \\
\text { of Hatakejima } \\
\text { Surface }\end{array}$} & 99.5 & 0.5 & 99.5 & 0 & 0.5 & 99.5 & 0 & 1.5 & 98.5 & \\
\hline & 100 & 1.0 & 98.0 & 1.0 & 0.5 & 99.5 & 0 & 1.5 & 98.5 & 0 \\
\hline & 97.0 & 5.0 & 95.0 & 0 & 1.0 & 99.0 & 0 & 3.0 & 97.0 & \\
\hline & 99.0 & 6.0 & 94.0 & 0 & 1.0 & 99.0 & 0 & 4.5 & 95.5 & \\
\hline \multirow[t]{2}{*}{ Bottom (15) } & 99.5 & 5.5 & 94.5 & 0 & 0.5 & 99.5 & 0 & 5.0 & 95.0 & 1 \\
\hline & 95.0 & 17.5 & 82.5 & 0 & 2.5 & 97.5 & 0 & 7.5 & 92.5 & \\
\hline \multirow{4}{*}{$\begin{array}{l}\text { Water from } \\
\text { land side of } \\
\text { Hatakejima } \\
\text { Surface }\end{array}$} & 99.0 & 1.0 & 99.0 & 0 & 1.0 & 99.0 & 0 & 2.5 & 97.5 & \\
\hline & 98.5 & 2.0 & 97.5 & 0.5 & 2.0 & 98.0 & 0 & 2.0 & 98.0 & 0 \\
\hline & 99.5 & 7.0 & 93.0 & 0 & 4.0 & 96.0 & 0 & 3.5 & 96.5 & \\
\hline & 99.0 & 1.5 & 98.0 & 0.5 & 2.0 & 98.0 & 0 & 3.0 & 97.0 & \\
\hline \multirow[t]{2}{*}{ Bottom (7) } & 99.5 & 2.0 & 97.5 & 0.5 & 1.5 & 98.5 & 0 & 2.5 & 97.5 & 1 \\
\hline & 93.0 & 12.0 & 87.0 & 1.0 & 3.5 & 96.5 & 0 & 4.5 & 95.9 & \\
\hline \multirow{3}{*}{$\begin{array}{l}\text { Sea water from } \\
\text { Tsunashirazu } \\
\text { cove } \\
\text { Surface }\end{array}$} & 99.0 & 1.5 & 98.5 & 0 & 1.5 & 98.5 & 0 & 5.0 & 95.0 & \\
\hline & 98.0 & 2.0 & 97.5 & 0.5 & 2.0 & 98.0 & 0 & 6.0 & 94.0 & 1 \\
\hline & 94.0 & 10.0 & 89.5 & 0.5 & 3.0 & 97.0 & 0 & 7.5 & 92.5 & \\
\hline \multirow{3}{*}{ Bottom (5) } & 98.0 & 4.0 & 96.0 & 0 & 2.5 & 97.5 & 0 & 2.0 & 98.0 & \\
\hline & 99.0 & 2.0 & 96.5 & 1.5 & 3.0 & 97.0 & 0 & 1.5 & 98.5 & 1 \\
\hline & 92.0 & 18.0 & 81.0 & 1.0 & 4.0 & 96.0 & 0 & 4.0 & 96.0 & \\
\hline
\end{tabular}


Ranking IV

\begin{tabular}{|c|c|c|c|c|c|c|c|c|c|}
\hline \multirow{3}{*}{$\begin{array}{l}\text { Location } \\
\qquad \text { (depth) }\end{array}$} & \multicolumn{3}{|c|}{ 1st cleavage $(75 \mathrm{~m})$} & \multicolumn{5}{|c|}{ Pluteus formation ( $48 \mathrm{~h}$ ) } & \multirow{3}{*}{$\begin{array}{l}\text { Remarks } \\
\text { grade }\end{array}$} \\
\hline & normal & 1 cell & $\begin{array}{l}\text { multi- } \\
\text { cell }\end{array}$ & normal & retard & $\begin{array}{l}\text { mal- } \\
\text { form }\end{array}$ & $\begin{array}{l}\text { pre- } \\
\text { pluteus }\end{array}$ & $\begin{array}{l}\text { dead } \\
\text { embryo }\end{array}$ & \\
\hline & \multicolumn{3}{|c|}{ (polyspermy) } & $\mathrm{N}$ & $\mathrm{R}$ & $P_{1}$ & $\mathrm{P}_{2}$ & $\mathrm{D}$ & \\
\hline \multirow{4}{*}{$\begin{array}{l}\qquad(\mathrm{m}) \\
\text { Running } \\
\text { sea water of } \\
\text { Laboratory }\end{array}$} & $\%$ & $\%$ & $\%$ & $\%$ & $\%$ & $\%$ & $\%$ & $\%$ & \\
\hline & 99.5 & 0.5 & & 97.5 & & 2.5 & & & \\
\hline & 99.0 & 1.0 & & 97.0 & & 3.0 & & & 0 \\
\hline & 97.0 & 3.0 & & 96.0 & & 4.0 & & & \\
\hline \multirow{3}{*}{$\begin{array}{l}\text { Water from } \\
\text { open sea side } \\
\text { of Hatakejima } \\
\text { Surface }\end{array}$} & 99.5 & & 0.5 & 93.5 & 1.0 & 5.5 & & & \\
\hline & 99.5 & 0.5 & & 94.5 & 2.0 & 3.0 & 0.5 & & 1 \\
\hline & 97.0 & 3.0 & & 92.5 & 0.5 & 7.0 & & & \\
\hline \multirow{3}{*}{ Bottom (15) } & 95.0 & 4.0 & 1.0 & 87.0 & 1.0 & 12.0 & & & \\
\hline & 95.0 & 5.0 & & 78.0 & & 21.5 & 0.5 & & 1 \\
\hline & 84.0 & 16.0 & & 69.0 & 4.0 & 25.5 & 1.5 & & \\
\hline \multirow{3}{*}{$\begin{array}{l}\text { Water from } \\
\text { land side of } \\
\text { Hatakejima } \\
\text { Surface }\end{array}$} & 98.5 & 1.0 & 0.5 & 96.5 & 0.5 & 2.5 & 0.5 & & \\
\hline & 98.5 & 1.5 & & 97.5 & & 2.0 & 0.5 & & 1 \\
\hline & 94.5 & 5.5 & & 91.5 & 0.5 & 6.0 & 2.0 & & \\
\hline \multirow{3}{*}{ Bottom (7) } & 98.5 & 1.5 & & 96.0 & & 4.0 & & & \\
\hline & 99.0 & 1.0 & & 93.5 & & 6.5 & & & 1 \\
\hline & 89.5 & 10.5 & & 85.0 & 1.5 & 12.0 & 1.5 & & \\
\hline \multirow{3}{*}{$\begin{array}{l}\text { Sea water from } \\
\text { Tsunashirazu } \\
\text { cove } \\
\text { Surface }\end{array}$} & 97.5 & 1.0 & 1.5 & 84.0 & 1.0 & 14.5 & 0.5 & & \\
\hline & 98.5 & 1.5 & & 82.0 & 2.0 & 16.0 & & & 1 \\
\hline & 90.5 & 9.5 & & 83.0 & 6.5 & 9.5 & 1.0 & & \\
\hline \multirow{3}{*}{ Bottom (5) } & 96.5 & 3.5 & & 94.5 & & 5.0 & 0.5 & & \\
\hline & 98.0 & 1.0 & 1.0 & 95.0 & 0.5 & 4.5 & & & 1 \\
\hline & 86.0 & 14.0 & & 83.0 & 3.0 & 12.0 & 2.0 & & \\
\hline
\end{tabular}


Table 17. Results of the May 30, 1988 experiment with eggs of Anthocidaris erassispina.

Wind; 0, Test water temperature; $22^{\circ} \mathrm{C}, 5$ mins. old sperms $3.5 \mathrm{hrs}$. old eggs. Ranking III 1985.

\begin{tabular}{|c|c|c|c|c|c|c|c|c|c|c|}
\hline \multirow{3}{*}{$\begin{array}{l}\text { Location } \\
\qquad \text { (depth) }\end{array}$} & \multicolumn{9}{|c|}{ Time after insemination } & \multirow{3}{*}{$\begin{array}{l}\text { Degree of } \\
\text { inhibitory } \\
\text { effect }\end{array}$} \\
\hline & \multirow{2}{*}{ 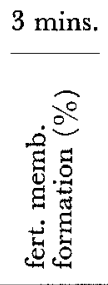 } & \multicolumn{3}{|c|}{60 mins. } & \multicolumn{3}{|c|}{$20 \mathrm{hrs}$. } & \multicolumn{2}{|c|}{36 hrs. } & \\
\hline & & 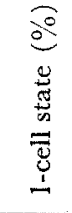 & 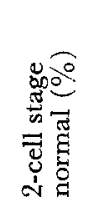 & 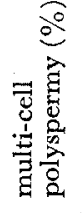 & 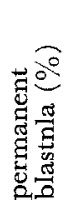 & 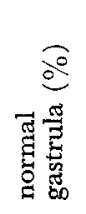 & 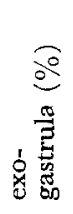 & 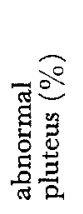 & 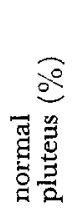 & \\
\hline \multicolumn{11}{|l|}{$(\mathrm{m})$} \\
\hline \multirow{3}{*}{$\begin{array}{l}\text { Running } \\
\text { sea water of } \\
\text { Laboratory }\end{array}$} & 94.5 & 6.0 & 93.0 & 1.0 & 1.5 & 98.5 & 0 & 2.5 & 97.5 & \\
\hline & 92.0 & 9.0 & 91.0 & 0 & 1.0 & 99.0 & 0 & 3.0 & 97.0 & 0 \\
\hline & 93.0 & 8.0 & 92.0 & 0 & 1.5 & 98.5 & 0 & 1.5 & 98.5 & \\
\hline \multirow{4}{*}{$\begin{array}{l}\text { Water from } \\
\text { open sea side } \\
\text { of Hatakejima } \\
\text { Surface }\end{array}$} & 91.5 & 8.5 & 90.0 & 1.5 & 2.5 & 97.5 & 0 & 2.5 & 97.5 & \\
\hline & 92.0 & 8.5 & 91.5 & 0 & 1.0 & 99.0 & 0 & 1.5 & 98.5 & 0 \\
\hline & 93.0 & 7.5 & 92.5 & 0 & 1.5 & 98.5 & 0 & 1.5 & 98.5 & \\
\hline & 87.0 & 13.5 & 86.5 & 0 & 3.5 & 96.5 & 0 & 1.5 & 98.5 & \\
\hline \multirow[t]{2}{*}{ Bottom (15) } & 88.0 & 13.0 & 87.0 & 0 & 5.5 & 94.5 & 0 & 3.0 & 97.0 & 1 \\
\hline & 90.0 & 11.0 & 89.0 & 0 & 5.0 & 95.0 & 0 & 2.0 & 98.0 & \\
\hline \multirow{4}{*}{$\begin{array}{l}\text { Water from } \\
\text { land side of } \\
\text { Hatakejima } \\
\text { Surface }\end{array}$} & 86.5 & 16.0 & 84.0 & 0 & 4.0 & 96.0 & 0 & 2.5 & 97.5 & \\
\hline & 86.0 & 15.0 & 85.0 & 0 & 3.5 & 96.5 & 0 & 3.0 & 97.0 & 1 \\
\hline & 85.0 & 16.5 & 83.5 & 0 & 2.5 & 97.5 & 0 & 3.5 & 96.5 & \\
\hline & 80.5 & 21.5 & 78.5 & 0 & 7.0 & 93.0 & 0 & 3.5 & 96.5 & \\
\hline \multirow[t]{2}{*}{ Bottom (7) } & 83.0 & 18.5 & 81.5 & 0 & 3.5 & 96.5 & 0 & 4.0 & 96.0 & 1 \\
\hline & 81.5 & 20.0 & 80.0 & 0 & 3.0 & 97.0 & 0 & 4.5 & 95.5 & \\
\hline \multirow{3}{*}{$\begin{array}{l}\text { Sea water from } \\
\text { Tsunashirazu } \\
\text { cove } \\
\text { Surface }\end{array}$} & 84.0 & 16.5 & 83.5 & 0 & 2.0 & 98.0 & 0 & 3.0 & 97.0 & \\
\hline & 85.5 & 16.0 & 84.0 & 0 & 6.0 & 94.0 & 0 & 3.5 & 96.6 & 1 \\
\hline & 83.0 & 17.5 & 82.5 & 0 & 6.5 & 83.5 & 0 & 5.0 & 95.0 & \\
\hline \multirow{3}{*}{ Bottom (5) } & 80.0 & 25.0 & 75.0 & 0 & 8.5 & 91.5 & 0 & 3.5 & 96.5 & \\
\hline & 81.5 & 20.0 & 80.0 & 0 & 6.5 & 93.5 & 0 & 4.5 & 95.5 & 1 \\
\hline & 79.5 & 21.5 & 78.5 & 0 & 6.0 & 94.0 & 0 & 6.0 & 94.0 & \\
\hline
\end{tabular}


Ranking IV

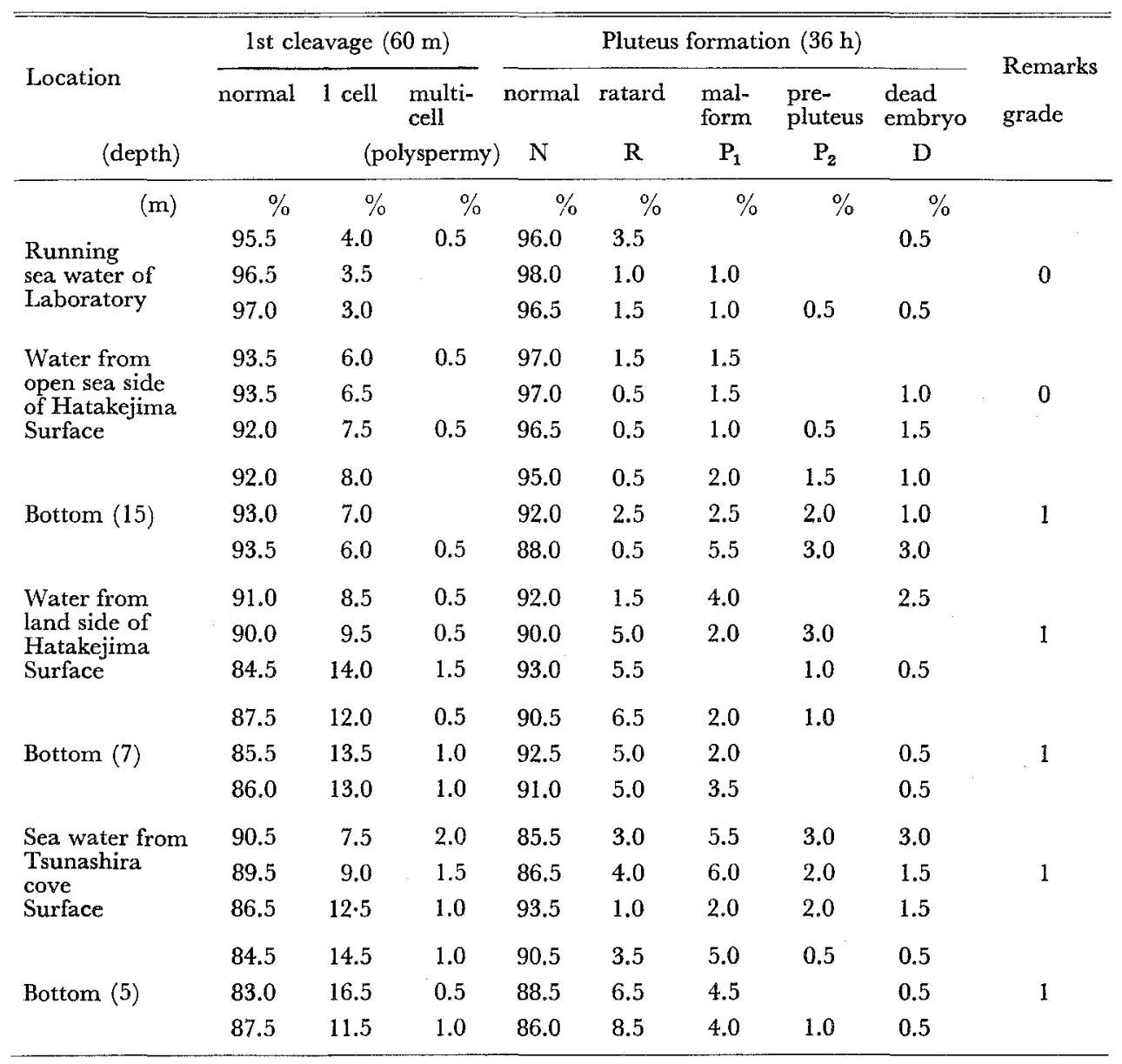


Table 18. Results of the July 19, 1988 experiment with eggs of Anthocidaris crassispina.

Wind; 0, Test water temperature; $26^{\circ}$ C, 5 mins. old sperms, 3 hrs. old eggs. Ranking III 1985.

\begin{tabular}{|c|c|c|c|c|c|c|c|c|c|c|}
\hline \multirow{3}{*}{$\begin{array}{l}\text { Location } \\
\qquad \begin{array}{l}\text { (depth) }\end{array}\end{array}$} & \multicolumn{9}{|c|}{ Time after insemination } & \multirow{3}{*}{$\begin{array}{l}\text { Degree of } \\
\text { inhibitory } \\
\text { effect }\end{array}$} \\
\hline & \multirow{2}{*}{$\frac{3 \text { mins. }}{\text { O̊㇒ }}$} & \multicolumn{3}{|c|}{60 mins. } & \multicolumn{3}{|c|}{$18 \mathrm{hrs}}$. & \multicolumn{2}{|c|}{36 hrs. } & \\
\hline & & 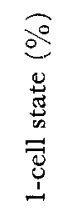 & 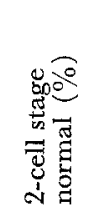 & 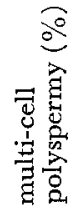 & 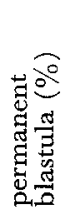 & 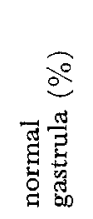 & 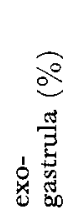 & 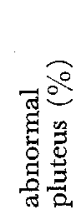 & 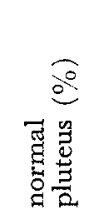 & \\
\hline \multicolumn{11}{|l|}{$(\mathrm{m})$} \\
\hline \multirow{3}{*}{$\begin{array}{l}\text { Running } \\
\text { sea water of } \\
\text { Laboratory }\end{array}$} & 97.0 & 3.5 & 96.5 & 0 & 0.5 & 99.5 & 0 & 0 & 100 & \\
\hline & 99.5 & 1.0 & 99.0 & 0 & 0.5 & 99.5 & 0 & 0.5 & 99.5 & 0 \\
\hline & 99.0 & 1.5 & 98.5 & 0 & 0 & 100 & 0 & 0.5 & 99.5 & \\
\hline \multirow{4}{*}{$\begin{array}{l}\text { Water from } \\
\text { open sea side } \\
\text { of Hatakejima } \\
\text { Surface }\end{array}$} & 98.5 & 2.0 & 98.0 & 0 & 0 & 100 & 0 & 0.5 & 99.5 & \\
\hline & 98.5 & 1.5 & 98.5 & 0 & 0.5 & 99.5 & 0 & 0.5 & 99.5 & 0 \\
\hline & 98.5 & 1.5 & 98.0 & 0 & 0.5 & 99.5 & 0 & 0.5 & 99.5 & \\
\hline & 96.5 & 4.0 & 96.0 & 0 & 1.5 & 98.5 & 0 & 1.0 & 99.0 & \\
\hline \multirow[t]{2}{*}{ Bottom (15) } & 98.0 & 2.0 & 98.0 & 0 & 1.0 & 99.0 & 0 & 1.5 & 98.5 & 0 \\
\hline & 98.0 & 2.5 & 97.5 & 0 & 1.0 & 99.0 & 0 & 2.0 & 98.0 & \\
\hline \multirow{4}{*}{$\begin{array}{l}\text { Water from } \\
\text { land side of } \\
\text { Hatakejima } \\
\text { Surface }\end{array}$} & 98.5 & 2.0 & 98.0 & 0 & 4.5 & 95.5 & 0 & 7.0 & 93.0 & \\
\hline & 99.0 & 1.5 & 98.5 & 0 & 8.5 & 91.5 & 0 & 10.5 & 89.5 & 1 \\
\hline & 98.0 & 2.5 & 97.5 & 0 & 7.0 & 93.0 & 0 & 9.5 & 90.5 & \\
\hline & 98.0 & 2.5 & 97.5 & 0.5 & 3.0 & 97.0 & 0 & 2.5 & 97.5 & \\
\hline \multirow[t]{2}{*}{ Bottom (7) } & 98.0 & 2.0 & 98.0 & 0 & 3.5 & 96.5 & 0 & 3.0 & 97.0 & 0 \\
\hline & 98.5 & 2.5 & 97.5 & 0 & 2.5 & 97.5 & 0 & 3.0 & 97.0 & \\
\hline \multirow{4}{*}{$\begin{array}{l}\text { Sea water from } \\
\text { Tsunashirazu } \\
\text { cove } \\
\text { Surface }\end{array}$} & 95.0 & 8.5 & 91.5 & 0 & 2.5 & 97.5 & 0 & 3.0 & 97.0 & \\
\hline & 98.5 & 3.0 & 97.0 & 0 & 5.0 & 95.0 & 0 & 4.5 & 95.5 & 1 \\
\hline & 97.5 & 3.5 & 96.5 & 0 & 4.0 & 96.0 & 0 & 4.0 & 96.0 & \\
\hline & 98.0 & 2.0 & 98.0 & 0 & 3.5 & 96.5 & 0 & 3.5 & $96 \cdot 5$ & \\
\hline \multirow[t]{2}{*}{ Bottom (5) } & 98.0 & 2.5 & 97.5 & 0 & 4.0 & 96.0 & 0 & 3.0 & 97.0 & 1 \\
\hline & 95.0 & 6.5 & 93.5 & 0 & 5.5 & 94.5 & 0 & 4.0 & 96.0 & \\
\hline
\end{tabular}


Ranking IV

\begin{tabular}{|c|c|c|c|c|c|c|c|c|c|}
\hline \multirow[b]{2}{*}{$\begin{array}{l}\text { Location } \\
\qquad(\text { depth })\end{array}$} & \multicolumn{3}{|c|}{ 1st cleavage $(60 \mathrm{~m})$} & \multicolumn{5}{|c|}{ Pluteus formation $(36 \mathrm{~h})$} & \multirow{2}{*}{$\begin{array}{l}\text { Remarks } \\
\text { grade }\end{array}$} \\
\hline & normal & $\begin{array}{l}1 \text { cell } \\
\quad(\mathrm{pc}\end{array}$ & $\begin{array}{l}\text { multi- } \\
\text { cell } \\
\text { lyspermy) }\end{array}$ & $\begin{array}{l}\text { normal } \\
\mathrm{N}\end{array}$ & $\begin{array}{c}\text { retard } \\
\mathbf{R}\end{array}$ & $\begin{array}{c}\text { mal- } \\
\text { form } \\
P_{1}\end{array}$ & $\begin{array}{l}\text { pre- } \\
\text { pluteus } \\
\mathbf{P}_{2} \\
\end{array}$ & $\begin{array}{l}\text { dead } \\
\text { embryo } \\
\text { D }\end{array}$ & \\
\hline \multirow{4}{*}{$\begin{array}{l}\text { Running } \\
\text { sea water of } \\
\text { Laboratory }\end{array}$} & $\%$ & $\%$ & $\%$ & $\%$ & $\%$ & $\%$ & $\%$ & & \\
\hline & 98.0 & 2.0 & & 99.0 & 1.0 & & & & \\
\hline & 99.0 & 0.5 & 0.5 & 99.5 & 0.5 & & & & 0 \\
\hline & 99.5 & 0.5 & & 99.0 & & 1.0 & & & \\
\hline \multirow{3}{*}{$\begin{array}{l}\text { Water from } \\
\text { open sea side } \\
\text { of Hatakejima } \\
\text { Surface }\end{array}$} & 98.5 & 1.5 & & 96.0 & & 2.0 & 2.0 & & \\
\hline & 98.5 & 0.5 & 1.0 & 98.5 & & 1.5 & & & 0 \\
\hline & 99.0 & 1.0 & & 99.5 & & 0.5 & & & \\
\hline \multirow{3}{*}{ Bottom (15) } & 96.5 & 3.5 & & 96.5 & 0.5 & 3.0 & & & \\
\hline & 97.0 & 2.0 & 1.0 & 96.5 & 1.5 & 2.0 & & & 0 \\
\hline & 98.5 & 1.5 & & 98.0 & 1.0 & 1.0 & & & \\
\hline \multirow{3}{*}{$\begin{array}{l}\text { Water from } \\
\text { land side of } \\
\text { Hatakejima } \\
\text { Surface }\end{array}$} & 98.0 & 2.0 & & 86.5 & 4.0 & 9.5 & & & \\
\hline & 98.5 & 1.5 & & 93.0 & 1.0 & 6.0 & & & 1 \\
\hline & 98.0 & 2.0 & & 91.0 & 1.5 & 7.5 & & & \\
\hline \multirow{3}{*}{ Bottom $(7)$} & 99.0 & 1.0 & & 96.5 & 3.0 & 0.5 & & & \\
\hline & 98.0 & 1.5 & 0.5 & 95.0 & 0.5 & 4.0 & 0.5 & & 0 \\
\hline & 99.0 & 1.0 & & 95.5 & 4.0 & 0.5 & & & \\
\hline \multirow{3}{*}{$\begin{array}{l}\text { Sea water from } \\
\text { Tsunashirazu } \\
\text { cove } \\
\text { Surface }\end{array}$} & 98.0 & 2.0 & & 90.5 & 1.0 & 4.5 & 4.0 & & \\
\hline & 96.5 & 3.0 & 0.5 & 88.5 & 4.5 & 3.5 & 3.5 & & 1 \\
\hline & 98.0 & 2.0 & & 90.0 & 2.0 & 4.0 & 4.0 & & \\
\hline \multirow{3}{*}{ Bottom (5) } & 98.0 & 2.0 & & 90.0 & 4.0 & 3.0 & 3.0 & & \\
\hline & 98.0 & 2.0 & & 89.0 & 4.0 & 3.5 & 3.5 & & 1 \\
\hline & 96.0 & 3.5 & 0.5 & 90.0 & 3.5 & 4.5 & 2.0 & & \\
\hline
\end{tabular}


Table 19. Results of the Aug. 19, 1988 experiment with eggs of Anthocidaris crassispina.

Wind; 0, Test water temperature; $27^{\circ} \mathrm{C}, 5$ mins. old sperms, 3 hrs. old eggs. Ranking III 1985.

\begin{tabular}{|c|c|c|c|c|c|c|c|c|c|c|}
\hline \multirow{3}{*}{$\begin{array}{l}\text { Location } \\
\begin{array}{l}\text { (depth) }\end{array} \\
\text { (des }\end{array}$} & \multicolumn{9}{|c|}{ Time after insemination } & \multirow{3}{*}{$\begin{array}{l}\text { Degree of } \\
\text { inhibitory } \\
\text { effect }\end{array}$} \\
\hline & \multirow{2}{*}{$\frac{3 \text { mins. }}{\text { 呑 }}$} & \multicolumn{3}{|c|}{60 mins. } & \multicolumn{3}{|c|}{$18 \mathrm{hrs}}$. & \multicolumn{2}{|c|}{36 hrs. } & \\
\hline & & 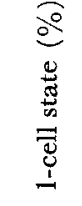 & 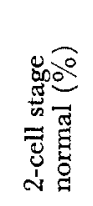 & 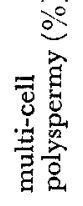 & 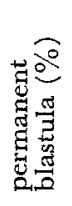 & 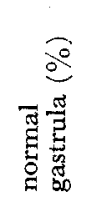 & 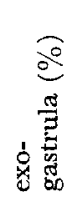 & 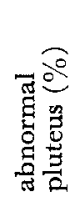 & 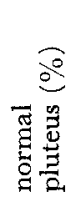 & \\
\hline \multicolumn{11}{|l|}{$(\mathrm{m})$} \\
\hline \multirow{3}{*}{$\begin{array}{l}\text { Running } \\
\text { sea water of } \\
\text { Laboratory }\end{array}$} & 97.5 & 3.5 & 96.5 & 0 & 1.0 & 99.0 & 0 & 2.0 & 98.0 & \\
\hline & 96.5 & 5.0 & 95.0 & 0 & 1.5 & 98.5 & 0 & 2.0 & 98.0 & 0 \\
\hline & 98.0 & 3.0 & 97.0 & 0 & 1.5 & 98.5 & 0 & 1.5 & 98.5 & \\
\hline \multirow{4}{*}{$\begin{array}{l}\text { Water from } \\
\text { open sea side } \\
\text { of Hatakejima } \\
\text { Surface }\end{array}$} & 96.0 & 4.5 & 95.5 & 0 & 1.0 & 99.0 & 0 & 2.0 & 98.0 & \\
\hline & 97.0 & 4.0 & 96.0 & 0 & 1.0 & 99.0 & 0 & 1.0 & 99.0 & 0 \\
\hline & 98.0 & 3.5 & 96.5 & 0 & 1.5 & 98.5 & 0 & 1.0 & 99.0 & \\
\hline & 92.5 & 9.0 & 91.0 & 0 & 2.0 & 98.0 & 0 & 3.5 & 96.5 & \\
\hline \multirow[t]{2}{*}{ Bottom (15) } & 91.0 & 10.5 & 89.5 & 0 & 2.5 & 97.5 & 0 & 2.0 & 98.0 & 0 \\
\hline & 93.0 & 9.5 & 90.5 & 0 & 2.5 & 97.5 & 0 & 3.5 & 96.5 & \\
\hline \multirow{4}{*}{$\begin{array}{l}\text { Water from } \\
\text { land side of } \\
\text { Hatakejima } \\
\text { Surface }\end{array}$} & 91.0 & 10.5 & 89.5 & 0 & 5.0 & 95.0 & 0 & 8.5 & 91.5 & \\
\hline & 89.5 & 12.0 & 88.0 & 0 & 6.0 & 94.0 & 0 & 8.0 & 92.0 & 1 \\
\hline & 88.0 & 13.5 & 86.5 & 0 & 4.0 & 96.0 & 0 & 7.0 & 93.0 & \\
\hline & 85.5 & 17.0 & 83.0 & 0 & 3.5 & 96.5 & 0 & 12.5 & 87.5 & \\
\hline \multirow[t]{2}{*}{ Bottom (7) } & 83.0 & 18.5 & 81.5 & 0 & 6.0 & 94.0 & 0 & 13.5 & 96.5 & 2 \\
\hline & 81.5 & 20.0 & 80.0 & 0 & 5.0 & 95.0 & 0 & 12.0 & 88.0 & \\
\hline \multirow{3}{*}{$\begin{array}{l}\text { Sea water from } \\
\text { Tsunashirazu } \\
\text { cove } \\
\text { Surface }\end{array}$} & 41.0 & 60.0 & 40.0 & 0 & & & & & & \\
\hline & 40.0 & 60.5 & 39.5 & 0 & & & & & & 5 \\
\hline & 38.5 & 63.5 & 36.5 & 0 & & & & & & \\
\hline \multirow{3}{*}{ Bottom (5) } & 69.5 & 31.5 & 68.5 & 0 & 7.0 & 93.0 & 0 & 9.0 & 91.0 & \\
\hline & 69.0 & 33.0 & 67.0 & 0 & 4.0 & 96.0 & 0 & 7.5 & 92.5 & 1 \\
\hline & 73.5 & 29.0 & 71.0 & 0 & 7.5 & 92.5 & 0 & 7.0 & 93.0 & \\
\hline
\end{tabular}


Ranking IV

\begin{tabular}{|c|c|c|c|c|c|c|c|c|c|}
\hline \multirow{3}{*}{$\begin{array}{l}\text { Location } \\
\qquad \text { (depth) }\end{array}$} & \multicolumn{3}{|c|}{ 1st cleavage $(60 \mathrm{~m})$} & \multicolumn{5}{|c|}{ Pluteus formation ( $36 \mathrm{~h}$ ) } & \multirow{3}{*}{$\begin{array}{l}\text { Remarks } \\
\text { grade }\end{array}$} \\
\hline & normal & 1 cell & $\begin{array}{l}\text { multi- } \\
\text { cell }\end{array}$ & normal & retard & $\begin{array}{l}\text { mal- } \\
\text { form }\end{array}$ & $\begin{array}{l}\text { pre- } \\
\text { pluteus }\end{array}$ & $\begin{array}{l}\text { dead } \\
\text { embryo }\end{array}$ & \\
\hline & \multicolumn{3}{|c|}{ (polyspermy) } & ) $N$ & $\mathrm{R}$ & $P_{1}$ & $\mathbf{P}_{2}$ & $\mathrm{D}$ & \\
\hline \multirow{4}{*}{$\begin{array}{l}\text { Running } \\
\text { sea water of } \\
\text { Laboratory }\end{array}$} & $\%$ & $\%$ & $\%$ & $\%$ & $\%$ & $\%$ & $\%$ & $\%$ & \\
\hline & 97.5 & 2.5 & & 96.5 & 2.5 & 1.0 & & & \\
\hline & 99.5 & 0.5 & & 96.0 & 2.5 & 1.5 & & & 0 \\
\hline & 99.0 & 1.0 & & 97.0 & 2.5 & 0.5 & & & \\
\hline \multirow{3}{*}{$\begin{array}{l}\text { Water from } \\
\text { open sea side } \\
\text { of Hatakejima } \\
\text { Surface }\end{array}$} & 98.5 & 1.0 & 0.5 & 97.0 & 1.0 & 2.0 & & & \\
\hline & 97.0 & 3.0 & & 98.0 & 1.5 & 0.5 & & & 0 \\
\hline & 96.5 & 3.5 & & 97.5 & 1.0 & 1.5 & & & \\
\hline \multirow{3}{*}{ Bottom (15) } & 97.0 & 3.0 & & 96.0 & 3.0 & 1.0 & & & \\
\hline & 94.5 & 5.5 & & 95.5 & 4.0 & 0.5 & & & 0 \\
\hline & 93.5 & 6.5 & & 95.5 & 3.0 & 1.5 & & & \\
\hline \multirow{3}{*}{$\begin{array}{l}\text { Water from } \\
\text { land side of } \\
\text { Hatakejima } \\
\text { Surface }\end{array}$} & 95.0 & 5.0 & & 86.0 & 14.0 & & & & \\
\hline & 95.5 & 4.5 & & 86.5 & 12.5 & 1.0 & & & 1 \\
\hline & 93.0 & 7.0 & & 81.0 & 16.5 & 2.5 & & & \\
\hline \multirow{3}{*}{ Bottom (7) } & 92.5 & 7.5 & & 63.5 & 28.0 & 8.5 & & & \\
\hline & 87.0 & 13.0 & & 72.0 & 21.0 & 7.0 & & & 2 \\
\hline & 84.5 & 15.5 & & 71.5 & 23.0 & 5.5 & & & \\
\hline \multirow{3}{*}{$\begin{array}{l}\text { Sea water from } \\
\text { Tsunashirazu } \\
\text { cove } \\
\text { Surface }\end{array}$} & 46.5 & 53.5 & & & & & & & \\
\hline & 39.0 & 61.0 & & & & & & & 3 \\
\hline & 42.5 & 57.5 & & & & & & & \\
\hline \multirow{3}{*}{ Bottom (5) } & 71.5 & 28.5 & & 82.0 & 11.0 & 7.0 & & & \\
\hline & 79.0 & 21.0 & & 87.5 & 7.5 & 5.0 & & & 1 \\
\hline & 76.5 & 23.5 & & 87.0 & 8.5 & 4.5 & & & \\
\hline
\end{tabular}


Table 20. Results of the Feb. 21, 1989 experiment with eggs of Hemicentrotus pulcherrimus. Wind; 0 . Test water temperature; $18^{\circ} \mathrm{C}$.

Ranking IV

\begin{tabular}{|c|c|c|c|c|c|c|c|c|c|}
\hline \multirow{2}{*}{ Location } & \multicolumn{3}{|c|}{ 1st cleavage $(75 \mathrm{~m})$} & \multicolumn{5}{|c|}{ Pluteus formation ( $48 \mathrm{~h}$ ) } & \multirow{3}{*}{$\begin{array}{l}\text { Remarks } \\
\text { grade }\end{array}$} \\
\hline & normal & 1 cell & $\begin{array}{l}\text { multi- } \\
\text { cell }\end{array}$ & normal & retard & $\begin{array}{l}\text { mal- } \\
\text { form }\end{array}$ & $\begin{array}{l}\text { pre- } \\
\text { pluteus }\end{array}$ & $\begin{array}{l}\text { dead } \\
\text { embryo }\end{array}$ & \\
\hline (depth) & & \multicolumn{2}{|c|}{ (polyspermy) } & $\mathrm{N}$ & $\mathbf{R}$ & $P_{1}$ & $\mathrm{P}_{2}$ & $\mathrm{D}$ & \\
\hline \multirow{4}{*}{$\begin{array}{l}\text { Running } \\
\text { sea water of } \\
\text { Laboratory }\end{array}$} & $\%$ & $\%$ & $\%$ & $\%$ & $\%$ & $\%$ & $\%$ & $\%$ & \\
\hline & 99.5 & 0.5 & & 96.5 & 2.5 & 1.0 & & & \\
\hline & 99.0 & 1.0 & & 93.5 & 3.5 & 3.0 & & & 0 \\
\hline & 99.5 & 0.5 & & 93.5 & 4.0 & 2.5 & & & \\
\hline \multirow{3}{*}{$\begin{array}{l}\text { Water from } \\
\text { open sea side } \\
\text { of Hatakejima } \\
\text { Surface }\end{array}$} & 97.5 & 2.0 & 0.5 & 94.5 & 3.0 & 2.5 & & & \\
\hline & 99.5 & 0.5 & & 94.0 & 3.5 & 2.5 & & & 0 \\
\hline & 99.0 & 1.0 & & 93.0 & 4.0 & 3.0 & & & \\
\hline \multirow{3}{*}{$\begin{array}{l}\text { Water from } \\
\text { land side of } \\
\text { Hatakejima } \\
\text { Surface }\end{array}$} & 97.0 & 3.0 & & 92.0 & 3.5 & 3.0 & 1.5 & & \\
\hline & 99.0 & 1.0 & & 94.0 & 3.0 & 2.5 & 0.5 & & 1 \\
\hline & 98.5 & 1.5 & & 91.0 & 4.0 & 3.5 & 1.5 & & \\
\hline \multirow{3}{*}{ Bottom (7) } & 97.5 & 2.5 & & 88.5 & 6.0 & 4.5 & 1.0 & & \\
\hline & 97.5 & 2.5 & & 87.5 & 5.5 & 6.5 & 0.5 & & 1 \\
\hline & 99.0 & 1.0 & & 84.5 & 7.5 & 7.0 & 1.0 & & \\
\hline \multirow{3}{*}{$\begin{array}{l}\text { Sea water from } \\
\text { Tsunashirazu } \\
\text { cove } \\
\text { Surface }\end{array}$} & 96.5 & 3.5 & & 89.0 & 4.5 & 5.5 & 1.0 & & \\
\hline & 98.0 & 2.0 & & 85.0 & 6.5 & 7.0 & 1.5 & & 1 \\
\hline & 98.5 & 1.5 & & 83.0 & 7.0 & 8.5 & 1.5 & & \\
\hline \multirow{3}{*}{ Bottom (5) } & 96.5 & 3.5 & & 84.5 & 7.0 & 6.0 & 2.5 & & \\
\hline & 97.5 & 2.5 & & 84.0 & 6.5 & 7.5 & 2.0 & & 1 \\
\hline & 99.0 & 1.0 & & 82.0 & 7.5 & 8.5 & 2.0 & & \\
\hline
\end{tabular}


Table 21. Results of the Apr. 5, 1989 experiment with eggs of Hemicentrotus pulcherrimus. Wind; 0 . Test water temperature; $17^{\circ} \mathrm{C}$.

Ranking IV

\begin{tabular}{|c|c|c|c|c|c|c|c|c|c|}
\hline \multirow{3}{*}{$\begin{array}{l}\text { Location } \\
\qquad \text { (depth) }\end{array}$} & \multicolumn{3}{|c|}{ Ist cleavage $(75 \mathrm{~m})$} & \multicolumn{5}{|c|}{ Pluteus formation ( $48 \mathrm{~h}$ ) } & \multirow{3}{*}{$\begin{array}{l}\text { Remarks } \\
\text { grade }\end{array}$} \\
\hline & normal & 1 cell & $\begin{array}{l}\text { multi- } \\
\text { cell }\end{array}$ & normal & retard & $\begin{array}{l}\text { mal- } \\
\text { form }\end{array}$ & $\begin{array}{l}\text { pre- } \\
\text { pluteus }\end{array}$ & $\begin{array}{l}\text { dead } \\
\text { embryo }\end{array}$ & \\
\hline & \multicolumn{3}{|c|}{ (polyspermy) } & ) $\mathrm{N}$ & $\mathrm{R}$ & $P_{1}$ & $\mathrm{P}_{2}$ & $\mathrm{D}$ & \\
\hline \multirow{4}{*}{$\begin{array}{l}\text { (m) } \\
\text { Running } \\
\text { sea water of } \\
\text { Laboratory }\end{array}$} & $\%$ & $\%$ & $\%$ & $\%$ & $\%$ & $\%$ & $\%$ & $\%$ & \multirow{4}{*}{0} \\
\hline & 99.5 & 0.5 & & 99.0 & 1.0 & & & & \\
\hline & 99.5 & 0.5 & & 99.5 & 0.5 & & & & \\
\hline & 99.0 & 1.0 & & 98.5 & 1.5 & & & & \\
\hline \multirow{3}{*}{$\begin{array}{l}\text { Water from } \\
\text { open sea side } \\
\text { of Hatakejima } \\
\text { Surface }\end{array}$} & 99.0 & 1.0 & & 98.5 & 1.5 & & & & \multirow{3}{*}{0} \\
\hline & 99.5 & 0.5 & & 97.0 & 2.0 & 1.0 & & & \\
\hline & 98.5 & 1.0 & 0.5 & 98.0 & 1.0 & 1.0 & & & \\
\hline \multirow{3}{*}{$\begin{array}{l}\text { Water from } \\
\text { land side of } \\
\text { Hatakejima } \\
\text { Surface }\end{array}$} & 99.0 & 1.0 & & 97.0 & 3.0 & & & & \multirow{3}{*}{0} \\
\hline & 98.5 & 1.5 & & 95.0 & 2.0 & 3.0 & & & \\
\hline & 98.5 & 1.0 & 0.5 & 96.0 & 2.0 & 2.0 & & & \\
\hline \multirow{3}{*}{ Bottom (7) } & 99.0 & 1.0 & & 95.0 & 5.0 & & & & \multirow{3}{*}{1} \\
\hline & 99.0 & 0.5 & 0.5 & 94.5 & 3.0 & 2.5 & & & \\
\hline & 98.0 & 1.5 & 0.5 & 91.5 & 6.5 & 2.0 & & & \\
\hline \multirow{3}{*}{$\begin{array}{l}\text { Sea water from } \\
\text { Tsunashirazu } \\
\text { cove } \\
\text { Surface }\end{array}$} & 99.0 & 1.0 & & 93.5 & 6.5 & & & & \multirow{3}{*}{1} \\
\hline & 98.5 & 1.5 & & 94.0 & 4.5 & 1.5 & & & \\
\hline & 98.0 & 1.5 & 0.5 & 92.5 & 6.5 & 1.0 & & & \\
\hline \multirow{3}{*}{ Bottom (5) } & 96.5 & 2.5 & 1.0 & & 31.5 & & 45.5 & 23.0 & \multirow{3}{*}{3} \\
\hline & 95.5 & 3.0 & 1.5 & & 48.5 & & 32.0 & 19.5 & \\
\hline & 96.0 & 3.5 & 0.5 & & 44.0 & 1.5 & 33.5 & 21.0 & \\
\hline
\end{tabular}


Table 22. Results of the July 5, 1989 experiment with eggs of Anthocidaris crassispina.

Wind; ; 0 . Test water temperature; $25^{\circ} \mathrm{C}$.

Ranking IV

\begin{tabular}{|c|c|c|c|c|c|c|c|c|c|}
\hline \multirow{3}{*}{$\begin{array}{l}\text { Location } \\
\qquad \text { (depth) }\end{array}$} & \multicolumn{3}{|c|}{ 1st cleavage $(60 \mathrm{~m})$} & \multicolumn{5}{|c|}{ Pluteus formation ( $36 \mathrm{~h}$ ) } & \multirow{3}{*}{$\begin{array}{l}\text { Remarks } \\
\text { grade }\end{array}$} \\
\hline & normal & 1 cell & & & & $\begin{array}{l}\text { mal- } \\
\text { form }\end{array}$ & $\begin{array}{l}\text { pre- } \\
\text { pluteus }\end{array}$ & $\begin{array}{l}\text { dead } \\
\text { embryo }\end{array}$ & \\
\hline & \multicolumn{3}{|c|}{ (polyspermy) } & $\mathrm{N}$ & $\mathrm{R}$ & $\mathbf{P}_{1}$ & $\mathrm{P}_{2}$ & $\mathrm{D}$ & \\
\hline \multirow{4}{*}{$\begin{array}{l}\quad(\mathrm{m}) \\
\text { Running } \\
\text { sea water of } \\
\text { Laboratory }\end{array}$} & $\%$ & $\%$ & $\%$ & $\%$ & $\%$ & $\%$ & $\%$ & $\%$ & \\
\hline & 97.0 & 3.0 & & 98.0 & 2.0 & & & & \\
\hline & 94.0 & 6.0 & & 96.0 & 3.5 & & 0.5 & & 0 \\
\hline & 98.0 & 2.0 & & 99.0 & 1.0 & & & & \\
\hline \multirow{3}{*}{$\begin{array}{l}\text { Water from } \\
\text { open sea side } \\
\text { of Hatakejima } \\
\text { Surface }\end{array}$} & 99.5 & 0.5 & & 99.5 & 0.5 & & & & \\
\hline & 95.0 & 5.0 & & 97.0 & 2.5 & & 0.5 & & 0 \\
\hline & 99.0 & 1.0 & & 98.5 & 1.5 & & & & \\
\hline \multirow{3}{*}{ Bottom (15) } & 97.5 & 2.5 & & 98.0 & 2.0 & & & & \\
\hline & 92.0 & 8.0 & & 97.0 & 2.0 & & 1.0 & & 0 \\
\hline & 96.0 & 4.0 & & 97.0 & 3.0 & & & & \\
\hline \multirow{3}{*}{$\begin{array}{l}\text { Water from } \\
\text { land side of } \\
\text { Hatakejima } \\
\text { Surface }\end{array}$} & 96.0 & 4.0 & & 97.0 & 2.0 & & 1.0 & & \\
\hline & 91.5 & 8.5 & & 95.0 & 3.0 & 1.0 & 1.0 & & 0 \\
\hline & 95.5 & 4.5 & & 96.0 & 2.5 & 0.5 & 1.0 & & \\
\hline \multirow{3}{*}{ Bottom (7) } & 91.0 & 9.0 & & 96.0 & 3.0 & 0.5 & 0.5 & & \\
\hline & 90.0 & 10.0 & & 93.0 & 4.0 & 1.0 & 2.0 & & 1 \\
\hline & 95.0 & 5.0 & & 95.0 & 3.5 & 0.5 & 1.0 & & \\
\hline \multirow{3}{*}{$\begin{array}{l}\text { Sea water from } \\
\text { Tsunashirazu } \\
\text { cove } \\
\text { Surface }\end{array}$} & 90.0 & 10.0 & & 94.5 & 5.0 & & 0.5 & & \\
\hline & 86.5 & 13.5 & & 90.0 & 6.5 & 1.0 & 2.5 & & 1 \\
\hline & 94.0 & 6.0 & & 93.0 & 5.5 & 0.5 & 1.0 & & \\
\hline \multirow{3}{*}{ Bottom (5) } & 91.0 & 9.0 & & 88.0 & 10.0 & 0.5 & 1.5 & & \\
\hline & 85.0 & 15.0 & & 83.0 & 12.0 & 1.5 & 3.5 & & 1 \\
\hline & 93.0 & 7.0 & & 87.0 & 11.0 & & 2.0 & & \\
\hline
\end{tabular}


Table 23. Results of the Aug. 30, 1989 experiment with eggs of Anthocidaris crassispina.

Wind; 0 . Test water temperature; $27^{\circ} \mathrm{C}$.

Ranking IV

\begin{tabular}{|c|c|c|c|c|c|c|c|c|c|}
\hline \multirow[b]{2}{*}{$\begin{array}{l}\text { Location } \\
\qquad \text { (depth) }\end{array}$} & \multicolumn{3}{|c|}{ 1st cleavage $(50 \mathrm{~m})$} & \multicolumn{5}{|c|}{ Pluteus formation ( $32 \mathrm{~h}$ ) } & \multirow{2}{*}{$\begin{array}{l}\text { Remarks } \\
\text { grade }\end{array}$} \\
\hline & normal & $\begin{array}{l}1 \text { cell } \\
\qquad(\mathrm{po}\end{array}$ & $\begin{array}{l}\text { multi- } \\
\text { cell } \\
\text { lyspermy) }\end{array}$ & $\begin{array}{l}\text { normal } \\
\mathrm{N}\end{array}$ & $\begin{array}{l}\text { retard } \\
\mathrm{R}\end{array}$ & $\begin{array}{l}\text { mal- } \\
\text { form } \\
P_{1}\end{array}$ & $\begin{array}{l}\text { pre- } \\
\text { pluteus } \\
\quad \mathrm{P}_{2}\end{array}$ & $\begin{array}{l}\text { dead } \\
\text { embryo } \\
\text { D }\end{array}$ & \\
\hline \multirow{4}{*}{$\begin{array}{l}\text { Running } \\
\text { sea water of } \\
\text { Laboratory }\end{array}$} & $\%$ & $\%$ & $\%$ & $\%$ & $\%$ & $\%$ & $\%$ & $\%$ & \\
\hline & 97.0 & 3.0 & & 98.0 & 1.0 & 0.5 & 0.5 & & \\
\hline & 98.5 & 1.5 & & 99.0 & 0.5 & 0.5 & & & 0 \\
\hline & 98.0 & 2.0 & & 97.5 & 1.5 & 1.0 & & & \\
\hline \multirow{3}{*}{$\begin{array}{l}\text { Water from } \\
\text { open sea side } \\
\text { of Hatakejima } \\
\text { Surface }\end{array}$} & 95.5 & 4.5 & & 93.0 & 2.5 & 2.5 & 2.0 & & \\
\hline & 94.0 & 6.0 & & 94.0 & 4.0 & 1.0 & 1.0 & & 1 \\
\hline & 96.5 & 3.5 & & 93.5 & 3.0 & 3.0 & 0.5 & & \\
\hline \multirow{3}{*}{ Bottom (15) } & 96.5 & 3.5 & & 97.0 & 2.5 & 0.5 & & & \\
\hline & 97.5 & 2.5 & & 98.0 & 1.0 & 1.0 & & & 0 \\
\hline & 99.0 & 1.0 & & 97.5 & 2.0 & 0.5 & & & \\
\hline \multirow{3}{*}{$\begin{array}{l}\text { Water from } \\
\text { land side of } \\
\text { Hatakejima } \\
\text { Surface }\end{array}$} & 92.5 & 7.5 & & 96.0 & 1.0 & 1.5 & 1.5 & & \\
\hline & 96.0 & 4.0 & & 94.0 & 3.0 & 2.0 & 1.0 & & 1 \\
\hline & 93.5 & 6.5 & & 94.5 & 3.5 & 1.0 & 1.0 & & \\
\hline \multirow{3}{*}{ Bottom (7) } & 92.5 & 7.5 & & 96.0 & 3.0 & 1.0 & & & \\
\hline & 95.0 & 5.0 & & 93.0 & 4.0 & 1.5 & 1.5 & & 1 \\
\hline & 91.5 & 8.5 & & 92.5 & 3.5 & 2.5 & 1.5 & & \\
\hline \multirow{3}{*}{$\begin{array}{l}\text { Sea water from } \\
\text { Tsunashirazu } \\
\text { cove } \\
\text { Surface }\end{array}$} & 92.0 & 8.0 & & 95.5 & 2.0 & 2.0 & 0.5 & & \\
\hline & 93.5 & 6.5 & & 92.0 & 4.5 & 1.5 & 2.0 & & 1 \\
\hline & 93.0 & 7.0 & & 93.5 & 4.0 & 1.5 & 1.0 & & \\
\hline \multirow{3}{*}{ Bottom (5) } & 93.5 & 6.5 & & 93.0 & 4.0 & 2.0 & 1.0 & & \\
\hline & 91.0 & 9.0 & & 90.5 & 8.0 & 1.0 & 0.5 & & 1 \\
\hline & 92.0 & 8.0 & & 89.0 & 10.0 & 0.5 & 0.5 & & \\
\hline
\end{tabular}

